\title{
Problemi di apprendimento di tre perifrasi fra spagnolo e italiano. Osservazioni acquisizionali e proposte pedagogiche.
}

\author{
Maria Vittoria Ambrosini / Paolo Della Putta ${ }^{2}$
}

Ricevuto: 22 dicembre 2020 / Modificato: 27 aprile 2021 / Accettato: 22 maggio 2021

Riassunto. Il contributo prende in considerazione alcuni problemi acquisizionali particolarmente ostici e persistenti nel processo di apprendimento dell'italiano da parte di discenti ispanofoni. Sono noti, in letteratura, i fenomeni di transfer additivo e persistente di strutture perifrastiche spagnole non presenti in italiano. È il caso di andare e venire + gerundio, strutture "gemelle" di ir e venir + gerundio: contemplate e frequenti, in spagnolo, in contesti diafasici anche bassi e trascurati, tali perifrasi, in italiano standard e neo standard, hanno perso molto "spazio" d'uso e pertengono, oggi, a varietà diafasiche alte della lingua. Per estar + gerundio e stare + gerundio - la terza coppia di perifrasi considerate in questo studio -, notiamo invece, in italiano, severe restrizioni aspettuali: estar + gerundio esprime sia l'aspetto progressivo che quello continuativo, mentre stare + gerundio si è specializzata solo nell'aspetto progressivo. Ne conseguono fenomeni di transfer spagnolo $\rightarrow$ italiano tipici, ben rintracciabili in realizzazioni come *sono stato lavorando tutta la notte. L'interpretazione psicolinguistica di tali problemi acquisizionali è basata sulla difficoltà di individuazione delle prove che possano permettere la ristrutturazione dell'interlingua: in questi tre casi sono prove negative indirette, ovvero la non apparenza delle strutture in determinati contesti o l'incongruenza con determinati valori aspettuali. Per lenire i problemi di transfer additivo delle tre perifrasi, in questo lavoro, dopo aver approfondito gli aspetti psicolinguistici di cui sopra, considereremo se e come le grammatiche pedagogiche di italiano per stranieri - in particolar modo ispanofoni - trattano queste discrepanze fra le due lingue. Proporremo infine alcuni accorgimenti pedagogici che possono essere utili per aiutare i discenti ispanofoni ad analizzare correttamente l'input italiano, così da lenire l'interferenza negativa fra le due lingue.

Parole chiave: perifrasi; transfer; prove negative indirette; grammatiche pedagogiche; glottodidattica.

\section{[en] Learning problems with three periphrases between Spanish and Italian. Acquisitional remarks and pedagogical proposals}

\footnotetext{
Abstract. This study takes into consideration some particularly difficult and persistent acquisitional problems that Spanish speakers who are learning Italian as a second language face. In literature, the phenomena of additive transfer of Spanish periphrastic structures which are not present in Italian is well documented. It is the case of andare and venire + gerund, which are "twin" structures of ir and venir + gerund. These are frequent in Spanish, even in low and neglected diaphasic contexts, but in standard and neo standard Italian, such periphrases display stronger contextual restrictions of use and are currently limited to high diaphasic varieties of Italian. For estar + gerund and stare + gerund, which are the third pair of periphrases analysed in this study, severe aspectual restrictions in Italian are noted: estar +

1 Universidad Autónoma de Barcelona (UAB). Departamento de Filología Francesa. 08193 Cerdanyola del Va1lès, Barcelona.

E-mail: ambromavi@yahoo.it

2 Università degli Studi di Torino, Dipartimento di Studi Umanistici, Via S. Ottavio 50, 10124 Torino.

E-mail: paoloantonio.dellaputta@unito.it
} 
gerund expresses both the progressive and the continuative aspect, while stare + gerund is specialised only in the progressive aspect. This subtle divergence gives rise to typical Spanish-Italian transfer phenomena which are clearly detectable in realisations such as *sono stato lavorando tutta la notte. The psycholinguistic interpretation of such acquisitional problems is based on the difficulty of identifying the evidence that can allow for the restructuring of the interlanguage. In these three cases it is indirect negative evidence, i.e., the non-appearance of the structures in certain contexts or the incongruence with certain aspectual values. In order to reduce the problems of additive transfer of the three periphrases, this paper will discern, after developing the psycholinguistic aspects mentioned above, whether the pedagogical grammars of Italian for foreigners, especially for Spanish speakers, deal with these discrepancies between the two languages and, if so, how. Finally, some pedagogical interventions that can be useful to help Spanish-speaking learners to correctly analyse the Italian input will be suggested to diminish the negative interference between the two languages.

Key words: periphrases; transfer; indirect negative evidence; pedagogical grammars; second language teaching.

Sommario: 1. Spagnolo e italiano: il labile confine tra affinità e trasferibilità 2. L'input e l'apprendente 2.1. L'input e le funzioni esecutive 3. Le tre perifrasi 3.1. Estar + gerundio / stare + gerundio 3.2. Ir + gerundio / andare + gerundio e venir + gerundio / venire + gerundio 3.3. Dati sul transfer delle perifrasi 4. Presenza e trattazione delle tre perifrasi nelle grammatiche di lingua italiana 5. Proposte pedagogiche 5.1. Considerare l'esistenza delle prove negative indirette 5.2. Manipolazione comparativa dell'input 5.3. Attività di ispirazione cognitiva 6. Conclusioni 7 Bibliografia.

Come citare: Ambrosini, M. V.; Della Putta, P. (2021): «Problemi di apprendimento di tre perifrasi fra spagnolo e italiano. Osservazioni acquisizionali e proposte pedagogiche», Cuadernos de Filología Italiana, 28, pp. 11-44.

\section{Spagnolo e italiano: il labile confine tra affinità e trasferibilità}

Le interlingue risultanti dal processo di acquisizione di una lingua obiettivo (LO) geneticamente e tipologicamente affine alla lingua madre (LM) presentano, sin dai loro esordi, un'organizzazione morfosintattica peculiare, di norma maggiormente articolata di quella rinvenibile nelle produzioni di apprendenti parlanti una LM non affine alla LO (Ringbom / Jarvis 2009; Díaz et al. 2007; Ringbom 2007, cap. 1). Questo fenomeno è facilmente osservabile nelle produzioni degli ispanofoni che apprendono l'italiano. Facilitati dall'oggettiva vicinanza strutturale dei due codici e da un atteggiamento psicotipologico generalmente piuttosto "disinvolto", gli hispanohablantes tentano - precocemente - di produrre categorie grammaticali complesse con mezzi morfosintattici articolati (Schmidt 1994: 196) ancorché, spesso, non pienamente target (cfr. discussione, estesa all'intera famiglia romanza, in Benazzo / Andorno 2017). Sin dai primi rilievi, le interlingue di questi apprendenti mostrano, dunque, proprietà delle varietà intermedie, scavalcando così alcune caratteristiche delle varietà pre-basica e basica (Schmid 1994: 80; Vietti 2005: 99-110). Vi è quindi consenso nel definire gli ispanofoni «facilitati dalla vicinanza strutturale» (Vietti 2005: 106) della loro LM all'italiano, tanto che è consuetudine, in percorsi di apprendimento guidato, inserire tali studenti in classi a ritmo veloce, spesso valutandoli come dei false beginners a cui dedicare accorgimenti didattici particolari.

La facilità in oggetto è stata molto efficacemente definita da Carrera Díaz (1979) come «engañosa»: essa è, infatti, sia una risorsa sia un impedimento per l'apprendimento, ed è un fenomeno da tenere "sotto controllo" in termini didattici. Se, da 
un lato - soprattutto nelle prime tappe del processo acquisizionale -, essa stimola la familiarizzazione con l'italiano, favorendo un'assimilazione piuttosto rapida delle sue proprietà morfosintattiche e permettendo una sua tendenziale comprensione (cfr. riflessioni in Rosi 2020), dall'altro essa può condurre a un'eccessiva assunzione di somiglianza tra i due idiomi, in termini sia di forme sia di norme di funzionamento; ciò può indurre rallentamenti o criticità nel processo di apprendimento, che è sovente costellato da transfer negativi tendenti alla fossilizzazione (Myers 2004: 102).

Analizzando nel dettaglio le interlingue degli ispanofoni, notiamo che presentano sia semplificazioni sia complessificazioni (Schmidt 1994: 208) della morfosintassi dell'italiano. Se, da un lato, si rilevano fenomeni di regolarizzazione dei paradigmi e di riduzione dell'allomorfia, comuni ad altri alloglotti, dall'altro lato è ben testimoniata, in letteratura, l'inserzione di strutture e/o funzioni dello spagnolo assenti in italiano. Ne risultano dunque fenomeni di erronea o inutile complessificazione di alcune aree della LO (Schmidt 1994: 210), alcuni dei quali, seguendo un approccio detection-based (Jarvis 2012) allo studio del transfer $^{3}$, elenchiamo senza pretesa di esaustività qui di seguito: l'accusativo preposizionale in frasi a ordine soggetto-verbo-oggetto (Della Putta 2018 inter alia), come in:

1) ho visto a Maria;

frequenti occorrenze di ridondanza pronominale (Schmidt 1994: 209), come in:

2) lei lo ha invitato a lui;

un uso generico e sovraesteso della perifrasi andare a + infinito in contesti e registri che, in italiano, non ne contemplano la presenza (Della Putta 2016; Morgana / Zaffaroni 2011), come in:

3) l'anno prossimo vado a essere ingegnere meccanico;

il ricorso a perifrasi costituite da verbi di movimento + gerundio in varietà colloquiali (Schmidt 1994: 197; Ambrosini 2018, 2020), come in:

4) c’è el signore che va mangiando un banano;

la mappatura di valori aspettuali continuativi sulla perifrasi stare + gerundio (Della Putta / Strik Lievers 2020; Ambrosini 2020), come in:

5) sono stato studiando tutta la notte.

Queste addizioni erronee avvengono per un eccesivo affidamento alla strategia della congruenza (Schmidt 1994) fra i due idiomi, che dona agli ispanofoni la convinzione di poter esprimere categorie grammaticali anche complesse (come valori aspettuali meno comuni o la marcatura di relazioni semantiche non prototipiche) tramite realizzazioni morfosintattiche tipiche della loro LM ma non pertinenti all' $\mathrm{i}$ -

L'approccio detection-based al transfer è così definito da Jarvis (2012: 1): «the detection of language-use patterns that are characteristic and distinctive of learners from specific L1 backgrounds». 
taliano. Si è infatti soliti dire che i problemi di apprendimento di una LO strettamente affine alla LM non risiedano nella (ri)costruzione di nuove categorie grammaticali quanto, piuttosto, nella corretta messa in grammatica di categorie affini alle due lingue (cfr. Amenós-Pons et al. 2017 per un'interpretazione generativista del problema). Secondo Corder (1984), infatti, gli apprendenti di una LO tipologicamente e geneticamente affine alla LM poggiano le loro ipotesi interlinguistiche sulle caratteristiche di quest'ultima, via via adattandole e modificandole sulla base dei dati provenienti dall'input, procedendo così in un processo di falsificazione e/o conferma delle ipotesi di funzionamento della LO create sul modello della LM. Si parla, in questo caso, di continuum di ristrutturazione: l'apprendente, grazie all'esposizione all'input e alla sua analisi, confuta o rinforza le "istintive" ipotesi di congruenza fra le due lingue. Nelle parole di Corder:

il continuum di ristrutturazione considera l'apprendente in un processo di graduale accomodamento della sua lingua materna per avvicinarsi progressivamente alla lingua obiettivo. [...] Il movimento lungo questo continuum equivale a una graduale sostituzione delle caratteristiche della lingua materna con le caratteristiche della lingua obiettivo. [...] Se si crede che un'altra lingua è [...] molto distante dalla nostra (ad es., cinese-italiano) è possibile optare per la strategia di ricreazione, cominciando da zero. [...] Al contrario, se invece si ritiene che la lingua obiettivo sia vicina alla nostra (ad es., spagnolo-italiano), possiamo preferire per una strategia di ristrutturazione. (Corder 1984: 53-67)

A simili conclusioni arriva Bailini dopo un minuzioso lavoro di analisi delle caratteristiche delle interlingue nate dall" "incontro" della coppia italiano-spagnolo:

el análisis paralelo de dos interlenguas cuyas características más sobresalientes se configuran a partir de la afinidad entre las respectivas lenguas maternas de los aprendentes permite arrojar luz sobre el papel determinante de la transferencia positiva y negativa y, también, afirmar que estas interlenguas, como sistemas, parecen ser el resultado de un proceso de reestructuración evolutiva en que la L1 es la plataforma sobre la cual se construye el aprendizaje de la L2. (Bailini 2016: 235)

Il presente lavoro è dedicato al fenomeno di complessificazione del sistema morfosintattico italiano da parte di apprendenti ispanofoni; in particolare, ci occuperemo del transfer di addizione superflua di tre perifrasi ${ }^{4}$ di uso molto comune in spagnolo: ir e venir + gerundio che, come vedremo, vengono impropriamente usate anche in registri informali (al pari di quanto accade in spagnolo) e stare + gerundio, su cui vengono mappati, oltre che al progressivo, valori aspettuali continuativi ${ }^{5}$, contrariamente a quanto accade in italiano. La sovraestensione di registri d'uso o di mappature aspettuali dalla LM alla LO può rivelarsi, per queste strutture, particolarmente

\footnotetext{
La RAE-ASALE (2009: 529) definisce in questo modo le perifrasi: «Se denominan perífrasis verbales las combinaciones sintácticas en las que un verbo auxiliar incide sobre un verbo auxiliado, principal o pleno, construido en forma no personal (es decir, en infinitivo, gerundio o participio), sin dar lugar a dos predicaciones distintas. El verbo auxiliar suele aparecer conjugado, pero puede no estarlo». Per un approfondimento più completo sulla/e definizione/i di perifrasi, rimandiamo a Bertinetto (1986: 331-347).

5 Nel presente contributo, abbiamo considerato la definizione di aspetto continuativo (da non confondere con aspetto continuo) proposta da García Fernández (2006: 46), che sarà illustrata in $\S 3.1$.
} 
insidiosa in quanto, se non opportunamente inquadrata in sede didattica, essa può generare una serie di prodotti linguistici che saranno, nel migliore dei casi, scarsamente fruibili o, nel peggiore di essi, del tutto agrammaticali, pur rimanendo tendenzialmente comprensibili, fattore, quest'ultimo, che ne può favorire la fossilizzazione. Nel prosieguo dell'articolo motiveremo da un punto di vista psicolinguistico la pervicacia del transfer di addizione, per poi presentare contrastivamente il caso delle perifrasi in oggetto. Infine, dopo aver passato in rassegna alcune ricerche che dimostrano l'alta fossilizzabilità degli esiti di questo transfer, estenderemo alcune considerazioni glottodidattiche che speriamo possano essere utili a lenire tali esiti.

\section{L'input e l'apprendente}

Come delineato poco sopra, il continuum di ristrutturazione prevede che l'apprendente raffronti e riveda le sue ipotesi di congruenza fra i due codici con le prove fornite dall'esposizione all'input della LO. Si è generalmente concordi nel vedere l'input come un fattore di assoluta necessità per una corretta evoluzione del processo di acquisizione di una $\mathrm{LO}^{6}$. Come ricorda Gass (1997: 1), infatti: «the concept of input is perhaps the single most important concept of second language acquisition. It is trivial to point out that no individual can learn a second language without input of some sort».

Lo stimolo linguistico innesca e mantiene attivo, nel tempo, lo sviluppo dell'interlingua. Tuttavia, alcuni fattori inerenti all'input come la salienza fonetica, la frequenza statistica, una relazione non univoca fra forma e funzione e il valore comunicativo degli elementi concorrono a variare la complessità di alcuni tratti della LO (Ellis 2006). A ciò si aggiungono variabili interindividuali quali le proprietà della LM, aspetti della personalità dell'apprendente e le sue possibilità di esposizione e uso della LO che restituiscono difficoltà acquisizionali intersoggettive diverse a parità di livello di complessità del fenomeno linguistico oggetto di apprendimento (Housen et al. 2019). Il ruolo della LM nella processazione dello stimolo linguistico è dirimente: essa funge da "filtro" cognitivo, a volte facilitante e a volte distraente, nella ricerca di prove che possano aiutare l'evoluzione interlinguistica (Ellis / Sagarra 2010). Si è inoltre generalmente concordi nell'asserire che l'input di LO fornisce diversi tipi di prove all'apprendente, suddivisibili in due macrocategorie: prove positive e prove negative. Delle prime fa parte tutto ciò che è percepibile nel significante, ovvero i fenomeni udibili e visibili direttamente dall'apprendente e "naturally occurring in native-speaker speech" (Bruton 2000: 121). Le prove positive possono essere manipolate dagli insegnanti, che possono potenziarne la salienza e la frequenza, usando per esempio tecniche di rafforzamento percettivo o di accrescimento della presenza di un elemento nel parlato o nello scritto. Le prove negative, invece, riguardano le forme e le espressioni linguistiche non presenti nella LO o in alcune sue varietà, e che dunque sono o completamente agrammaticali o proprie solo di alcuni contesti comunicativi. Come scrive Bruton (2000: 125), le prove negative si manifestano

Dobbiamo a Krashen le prime riflessioni sulla centralità dell'input e sulle condizioni ottimali per la sua processazione e per la sua trasformazione in intake. Il discorso sulle proprietà e sul valore dello stimolo linguistico, da allora, si è fatto molto rilevante, ed è impossibile affrontarlo in questa sede. Una valida e aggiornata testimonianza dell'ampiezza e della profondità di tale tema, e delle tante possibilità di esposizione allo stimolo della LO, è rintracciabile in Peters e Muñoz (2020), nella loro introduzione a un numero speciale di Studies in Second Language Acquisition dedicato proprio all'input multimodale nell'apprendimento linguistico. 
tramite «what is not said» e possono essere a loro volta dirette e indirette; le correzioni degli insegnanti sono prove negative dirette: esse catalizzano l'attenzione degli apprendenti su una produzione non target nella LO; le prove negative indirette, invece, equivalgono alla non apparenza nell' input di strutture non contemplate nella $\mathrm{LO}$, in toto o in alcune sue varietà. Un esempio di tal ultima categoria di prova, per tornare alla coppia linguistica qui considerata, è l'assenza, in italiano standard, della $a$ pre-oggetto specifico e animato in una frase a ordine canonico soggetto-verbo-oggetto. Uno studente ispanofono dovrebbe, in questo caso, ristrutturare correttamente la sua interlingua notando una prova negativa indiretta, ovvero la mancanza della $a$ pre-oggetto animato in frasi SVO; notare questo permetterebbe al discente di andare contro il modello della sua LM, che invece contempla l'accusativo preposizionale in tale contesto frasale (Della Putta 2018).

Gli apprendenti ispanofoni di italiano devono fare affidamento a entrambi i tipi di prove per accomodare la loro interlingua al modello dell'italiano. Tuttavia, una prova positiva è uno stimolo più forte e affidabile di una prova negativa indiretta: un elemento fisicamente presente nell'input ha un "corpo", dunque possiede un certo livello di salienza percettiva - ancorché, in qualche caso, minima - e risulta, quindi, più facilmente notabile e processabile di un elemento invisibile perché completamente assente.

\subsection{L'input e le funzioni esecutive}

Per rendere conto con più completezza del ruolo dell'input nel processo acquisizionale, occorre spostare ora la nostra argomentazione da una prospettiva eminentemente linguistica a una lettura più psicolinguistica. È a questo punto quindi necessario chiedersi come l'apprendente estrapoli le informazioni dallo stimolo e quali siano le facoltà mentali impiegate in questa operazione, in particolar modo in un contesto di lingue affini come quello a cui facciamo qui riferimento. In linea con gli approcci di natura cognitivo/funzionalista, che vedono coinvolti nel processo di apprendimento di una LO le stesse facoltà mentali reclutate per altre finalità, sia generiche sia di apprendimento (Ellis 2019), consideriamo in quanto segue il ruolo delle funzioni esecutive (FE) nel processo acquisizionale e la loro relazione con le diverse prove portate dall'input di una LO.

Le FE sono «processi mentali necessari a programmare, mettere in atto e portare a termine con successo un comportamento finalizzato a uno scopo» (Benso 2013: 56). In particolare, le FE sono deputate a: 1) avviare un'azione pianificata; 2) sostenere l'attenzione verso gli stimoli utili per portare a termine l'azione; 3) controllare, autoregolandoli, pensieri ed emotività distraenti; 4) inibire stimoli, esterni o interni, che possono essere distraenti o confondenti verso l'azione; 5) rielaborare le tracce mnestiche immagazzinando nuovi dati ed eventualmente sopprimendo quelli obsoleti o non funzionali al compimento dell'azione. Delle FE fanno parte tre ${ }^{7}$ importanti facoltà mentali: l'attenzione selettiva, la memoria di lavoro e il controllo inibitorio, che di norma si è avvezzi a considerare come facoltà distinte. Ci si è infatti spesso focalizzati, anche nella ricerca acquisizionale, sulle singole proprietà di ognuna di esse, anche se le più moderne prospettive integrate sull'attività mentale suggerisco-

Non c'è consenso su quante siano le facoltà mentali che effettivamente fanno parte delle FE. Per i nostri scopi ci limitiamo a citare le tre più ampiamente attestate; rimandiamo il lettore, per approfondimenti, a Benso (2013). 
no di studiarle come meccanismi interagenti, la cui contemporanea attivazione è funzionale al raggiungimento di azioni e scopi pianificati (Diamond 2013; Linck et al. 2020).

Volendo semplificare molto le cose, all'attenzione selettiva spetta il compito di catturare le prove date dall'input, in una costante attività di mediazione fra lo stimolo esterno e lo stimolo interno, sia psichico - motivazioni verso l'apprendimento e necessità contestuali - sia linguistico - alcune caratteristiche della LM faciliteranno la selezione di alcune caratteristiche di LO ma non di altre. La memoria di lavoro ha il compito di immagazzinare temporaneamente queste informazioni e di "mantenerle vive" affinché possano essere fruibili durante il compito in atto, permettendo così la loro trasferibilità alla memoria a lungo termine. Il controllo inibitorio, infine, fa sì che stimoli distraenti o antitetici non interferiscano con il compito in atto: è questo il caso della LM, che deve essere inibita durante la produzione della LO (Linck et al. 2020). In particolare, l'inibizione dell'attivazione di proprietà morfosintattiche "fini" della LM durante l'uso di una LO è un processo cognitivo complesso, ancor più quando le due lingue sono strettamente imparentate (Abutalebi et al. 2013; Jarvis et al. 2013). Inoltre, durante l'acquisizione di una LO simile alla LM, inibire l'attivazione di una regola della LM quando molte altre possono essere trasferite verso la LO senza incappare in problemi comunicativi è un'operazione mentale molto complessa; essa richiede, infatti, livelli di attenzione selettiva molto alti, tali da portare alla memoria di lavoro prove concrete di come i due sistemi divergono e di quali proprietà o tratti sia necessario inibire per evitarne il transfer.

Le FE sono maggiormente sensibili alle prove positive e alle prove negative dirette, che danno una concreta (ovvero percepibile perché presente nell'ambiente) indicazione su cosa c'è e su cosa è errato nella LO. I tratti dell'interlingua che, invece, devono essere ristrutturati sulla base di prove negative indirette e che, per venire a capo del transfer di addizione superflua (cfr. supra), non devono comparire in certi contesti interlinguistici, sono maggiormente impermeabili all'attività delle FE. In primo luogo, l'attenzione selettiva farà fatica a carpire "ciò che manca" nell'input: l'apprendente può soltanto fare affidamento sulla non apparenza di una forma nella (o in alcune varietà della) LO o di una funzione della LM la cui mappatura non è contemplata su una struttura formalmente simile della LO. La scarsa percettibilità di queste prove, studiata sia in ambito generativista che cognitivo/funzionalista (cfr. discussione in Robenhalt / Goldberg 2016), implica una difficile ristrutturazione delle conoscenze immagazzinate in memoria di lavoro: l'apprendente continuerà a usare il bagaglio informativo portato dalla sua LM e non riuscirà a riorganizzare le informazioni seguendo il modello della LO. Infine, in caso di prova negativa indiretta sappiamo che il controllo inibitorio richiede un maggior dispendio energetico/cognitivo per bloccare il transfer di una struttura o proprietà presente in LM ma assente in LO (Jarvis et al. 2013: 293). Nel caso di lingue simili, quindi, è più difficile confutare l'ipotesi di congruenza proprio quando l'apprendente ritiene di poter usare una struttura della sua LM non contemplata dalla LO: le FE faranno fatica ad analizzare correttamente l'input e a inibire le informazioni distraenti fornite dalla LM.

Si delinea così il quadro psicolinguistico sottostante ai problemi acquisizionali in cui gli studenti ispanofoni di italiano incappano quando devono limitare il transfer delle perifrasi da noi considerate. Essi devono notare che andare e venire + gerundio, le controparti italiane di ir e venir + gerundio, non compaiono nelle varietà colloquiali di italiano, a differenza di quanto accade in spagnolo, e che il loro uso è 
limitato a contesti e registri alti. Per estar + gerundio, invece, gli hispanohablantes devono notare che la perifrasi gemella, stare + gerundio, non può esprimere l'aspetto continuativo e che, conseguentemente, non può essere usata con i tempi composti. L'inibizione del transfer di queste perifrasi è dunque, per i motivi visti sopra, molto complessa e richiede attenzioni pedagogiche particolari.

\section{Le tre perifrasi}

Prendiamo ora in considerazione più da vicino le tre perifrasi oggetto di questo studio. Ne analizzeremo le proprietà in spagnolo, per poi proporre un'analisi contrastiva con le loro controparti italiane. Consideriamo dunque le seguenti tre coppie perifrastiche:

- $\quad$ estar + gerundio / stare + gerundio

- $\quad$ ir + gerundio / andare + gerundio

- venir + gerundio / venire + gerundio

\subsection{Estar + gerundio / stare + gerundio}

La perifrasi estar + gerundio può veicolare molteplici valori aspettuali, a seconda del contesto comunicativo all'interno del quale si inserisce; solo alcuni di questi valori, tuttavia, sono associabili anche alla sua (formalmente) corrispondente italiana stare + gerundio.

Secondo García Fernández (2006: 136), nei casi in cui il verbo ausiliare è un presente, un imperfetto, un futuro o un condizionale, la perifrasi estar + gerundio esprime aspetto progressivo, valore aspettuale che, secondo Camus Bergareche (2004: 539), caratterizza: «situaciones en las que se describe un instante concreto y no iterado en el desarrollo de una situación de cuyo inicio o final no se dice nada». Si tratta di un valore aspettuale che può essere espresso anche dalla perifrasi italiana stare + gerundio, come nei seguenti esempi:

6) Esto es precisamente uno de los principales cambios que quieren introducir en el nuevo Estatuto de Autonomía que en este momento están debatiendo los grupos parlamentarios. (Calleja 2006)

7) Sta dormendo profondamente (Bertinetto 1991: 132)

Tuttavia, se la perifrasi estar + gerundio presenta ausiliare in forma composta, il valore aspettuale da essa espresso non è più il progressivo ma il continuativo. García Fernández (2006: 46-48) dà la seguente definizione di aspetto continuativo:

el Tiempo del Foco abarca desde el inicio del Tiempo de la Situación hasta un punto interno de su desarrollo. La perífrasis $<$ llevar + gerundio $>$ expresa exactamente este significado aspectual; así en Juan lleva dos horas pintando la casa, el evento denotado por pintar la casa ha empezado hace dos horas y continúa en el momento de la enunciación sin que se predique su final. 
Esempi:

8) Ha estado durmiendo hasta ahora y necesita dormir más. (García Fernández 2006: 136)

9) Habian estado discutiendo hasta que había llegado el director, pero no se interrumpieron. (García Fernández 2006: 136)

Riteniamo raccomandabile considerare la distinzione tra aspetto continuo e aspetto continuativo, essenziale per potersi accostare a questo contributo secondo una corretta prospettiva. L'aspetto continuativo inquadra il processo azionale a partire dal suo punto iniziale; dello stesso processo, invece, non si esplicita - né si può evincere con chiarezza - il punto finale, che per tanto rimane sospeso. L'aspetto continuo, invece, rappresenta una delle tre manifestazioni dell'imperfetto (Bertinetto 1986: 139162), la quale considera un certo arco temporale interno del processo azionale (senza focalizzazione incidenziale su alcuno dei suoi punti specifici): dello stesso, quindi, risulterà sospeso non solo il limite finale (come nel caso dell'aspetto continuativo), ma anche il limite iniziale 8 .

Secondo Squartini (1998: 74-75), di fatto, il valore progressivo sarebbe l'unico valore aspettuale che può assumere la perifrasi italiana stare + gerundio, come risultato del processo di grammaticalizzazione che l'avrebbe portata ad assumere la sua tipizzazione attuale:

\section{LOCATIVITY $>$ DURATIVITY $>$ IMPERFECTIVE PROGRESSIVITY $>$ ? $[+$ ACTIONALITY] $>[-$ ACTIONALITY $]$ $[-\mathrm{ASPECT}]>[+\mathrm{ASPECT}]$}

Inizialmente, il verbo stare era maggiormente legato alla sua componente lessicale, essendo utilizzato fondamentalmente come verbo locativo. Successivamente, avrebbe perso parte della sua componente semantica, avvicinandosi ad una valenza aspettuale durativa: a partire da allora, avrebbe assunto un nuovo uso in forma imperfettiva, associandosi, all'interno della perifrasi, ad azioni in corso nel momento di enunciazione (aspetto progressivo). Con il passare del tempo, la perifrasi italiana si sarebbe quindi stabilizzata come perifrasi di carattere eminentemente progressivo. Come osserva Bertinetto (1997: 226-228):

Una caratteristica che oppone tra di loro le perifrasi delle tre lingue considerate $[$ stare + gerundio per l'italiano, estar + gerundio per lo spagnolo e be $+V$-ing form per l'inglese] è la vocazione rigorosamente imperfettiva del costrutto italiano. [...] Ai fini del nostro discorso è soprattutto importante notare che l'inglese e lo spagnolo si mostrano pienamente solidali, e in netto contrasto con l'italiano, in contesti come i seguenti $[\ldots]$ :

a. *Stamattina, Giulio stava continuamente cercando il suo quaderno durante la lezione.

b. Esta mañana Julio estaba buscando continuamente su cuaderno durante la clase.

\footnotetext{
Per ulteriori riferimenti in merito alla caratterizzazione dell'aspetto continuo e continuativo, vedasi anche Mar-
} tínez Atienza (2004: 346-377), García Fernández (2006: 45-47) e Montserrat i Buendia (2005: 54). 
c. This morning, Julius was continuously looking for his exercice-book during the class.

La caratteristica di questi enunciati sta nel fatto di presentare l'evento in rapporto ad un intervallo di riferimento, di durata tendenzialmente determinata - benché i suoi limiti non siano esattamente indicati - anziché in rapporto ad un singolo istante di riferimento (o, come si dice anche, "di focalizzazione"). Ciò corrisponde alle proprietà semantiche dell'aspetto continuo, piuttosto che a quelle dell'aspetto progressivo propriamente detto, com'è stato diffusamente argomentato in Bertinetto $(1986)^{9}$.

Con il consolidarsi, nel processo di grammaticalizzazione, di questa vocazione prettamente imperfettiva della perifrasi stare + gerundio, si sono profilate alcune delle sue principali divergenze rispetto alla corrispondente spagnola. L'ausiliare estar, ad esempio, ammette anche verbi ausiliati di natura risultativa o trasformativa (Vendler 1967: 22) - ossia atelici - cui imprime, nella combinazione perifrastica, una valenza durativo-continuativa che ne neutralizza la stessa telicità di base (Bertinetto 2000: 570-571):

\section{0) He estado preparando la cena/ pintando la pared todo el día}

L'ausiliare stare, dal canto suo, si conferma invece piuttosto restio a combinarsi con predicati telici. Un'altra conseguenza del carattere imperfettivo della perifrasi italiana, come si evince dall'esempio (5), si riflette direttamente sulla limitazione del paradigma dell'ausiliare stare, che in nessun caso può essere un tempo composto (Bertinetto 2000: 566-568). In un certo senso, ipotizza Squartini (1998: 75), nel castigliano attuale 1'ausiliare estar si trova nella tappa del processo di grammaticalizzazione precedente rispetto all'ausiliare italiano, in quanto accetta ancora la morfologia associata all'aspetto durativo-continuativo. Su un piano contrastivo, dunque, il raffronto tra estar + gerundio e stare + gerundio può dar luogo a due casi possibili:

A) Si constaterà una piena corrispondenza tra perifrasi italiana e perifrasi spagnola se (e solo se) il contesto aspettuale è progressivo, e dunque se entrambi gli ausiliari sono forme verbali semplici (non composte). Proponiamo due esempi:
11)
a. Cuando he entrado en su cuarto, María estaba durmiendo
b. Quando sono entrato nella sua camera, María stava dormendo

\footnotetext{
Tuttavia, come osserva lo stesso Bertinetto (1997: 236-238), esistono casi in cui il confine tra progressività e continuità si fa piuttosto labile: in questi casi, la distinzione tra - rispettivamente - monofocalizzaione e plurifocalizzazione sull'evento osservato viene meno, specie in presenza di modificatori che lo reinterpretino in chiave graduale-processuale: «La situazione stava/andava peggiorando sempre più» / «Stava sempre ideando e appendendo cartelli per annunciare la bontà dei prodotti...»/ «non sapevo però leggere le note, e ignoravo assolutamente cosa fosse la teoria musicale, se si esclude quel poco che stavo apprendendo a scuola durante l'ora di canto». L'autore osserva che anche i contatti con l'inglese o lo spagnolo, con perifrasi progressive tendenzialmente più adattabili a contesti durativi, avrebbero potuto incidere in tal senso. Per una più ampia documentazione relativamente a questi aspetti, vedasi anche Bertinetto (1986: 139-162; 2000: 559-604). Per ulteriori approfondimenti sullo sviluppo diacronico della perifrasi stare + gerundio, difficilmente illustrabile in modo esaustivo in questa sede, rimandiamo invece a Brianti (2000: 97-119; 2010: 351-362).
} 
12) a. Ahora no puedo ayudarte, estoy estudiando para el examen de mañana b. Non posso aiutarti adesso, sto studiando per l'esame di domani

B) Si constaterà invece una divergenza tra le due lingue coinvolte nel momento in cui la perifrasi spagnola designa un'azione continuativa, ricorrendo all'uso della perifrasi estar + gerundio con ausiliare in forma composta (uso non ammesso in italiano per la perifrasi stare + gerundio). Forniamo anche qui un paio di esempi:

13) a. Ha estado durmiendo hasta ahora y necesita dormir más (García Fernández 2006: 136)

b. * É stato dormendo fino ad ora e ha bisogno di dormire ancora

14) a. Habian estado discutiendo hasta que había llegado el director, pero no se interrumpieron (García Fernández 2006: 136)

b. * Erano stati discutendo finché era arrivato il direttore, ma non si interruppero

I risultati del transfer differiscono molto fra A e B. Nel primo caso l'esito è positivo in quanto vi è piena corrispondenza fra LM e LO sia nella forma che nella funzione, ovvero nel valore aspettuale progressivo mappato sulle perifrasi. In B, invece, l'apprendente estende il paradigma dell'ausiliare stare anche alle sue forme composte, alla pari di quanto fa spontaneamente, in LM, per l'ausiliare estar; in questa operazione leggiamo anche un'impropria associazione a livello funzionale, in quanto il valore aspettuale continuativo proprio di estar + gerundio viene trasferito a stare + gerundio.

\subsection{Ir + gerundio / andare + gerundio e venir + gerundio / venire + gerundio}

Le due coppie di perifrasi oggetto di analisi di questo paragrafo presentano un'evidente affinità formale a cui si contrappone, però, un'asimmetria di contesti e di frequenza d'uso, nonché una certa divergenza in termini di valori funzionali tra LM e LO. Proponiamo una panoramica generale per quanto riguarda, innanzitutto, i loro valori aspettuali.

Ir + gerundio può veicolare due aspetti distinti, continuo o continuativo. Secondo García Fernández (2006: 171-174), se la perifrasi è accompagnata da un complemento avverbiale (CA) che indica il momento in cui l'azione ha iniziato a svilupparsi, si parla di aspetto continuativo:

15) Voy aumentando la velocidad, desde que he visto la hora (García Fernández 2006: 174)

In assenza del CA che segnali l'inizio dell'azione, invece, la perifrasi ha valore aspettuale continuo:

16) Cuando vamos creciendo, el destino se complace en variarnos como si fuésemos de cera (Sánchez Montero 1993: 105) 
La perifrasi venir + gerundio, invece, presenta un valore intrinsecamente continuativo $^{10}$ anche nei casi in cui non è accompagnata da un CA che segnali l'inizio dell'azione, in quanto, come osserva Camus Bergareche (2004: 558): «A diferencia de las otras perífrasis que pueden expresar aspecto continuativo, venir + gerundio no tiene por qué ir acompañada siempre por el complemento adverbial que señala el comienzo de la acción. Este complemento, en efecto, puede ser implícito en la situación comunicativa, debido probablemente a las peculiaridades léxicas del auxiliar venir». Tuttavia, la presenza del CA tende a favorire maggiormente un'interpretazione di tipo continuativo dell'azione in oggetto, mentre la sua assenza ne favorisce piuttosto una di ordine continuo-abituale; si osservino, a titolo d'esempio, le frasi seguenti:

17) Las razones por las que se viene insistiendo en la peligrosidad de estos hábitos siguen siendo oscuras (Sánchez Montero 1993: 119)> (aspetto continuo-abituale) 18) La página editorial de este diario viene siendo muy crítica desde las elecciones. (Camus Bergareche 2004: 558) > (aspetto continuativo)

Per quanto concerne invece le perifrasi italiane andare + gerundio e venire + gerundio, è innanzitutto importante sottolineare che la maggior parte delle grammatiche di lingua italiana attualmente disponibili non cita né tratta queste due perifrasi, punto su cui torneremo in $\S 4$. Bertinetto (1991: 13-161) associa a entrambe la modalità aspettuale continua, al punto da riunirle sotto la denominazione unica di perifrasi continua ${ }^{11}$. Esempi:

19) Alfonso andava meditando sui propri guai (Bertinetto 1991: 144)

20) Le istituzioni si venivano orientando in senso sempre più democratico. (Sánchez Montero 1993: 118)

Tuttavia, come osserva Martínez Atienza (2017: 32), venire + gerundio può anch'essa esprimere modalità continuativa, in determinati contesti situazionali. Proponendo ora un raffronto tra le due perifrasi spagnole e le due perifrasi italiane in termini di struttura morfologica e di livello di grammaticalizzazione, possiamo osservare quanto segue.

A differenza di estar / stare + gerundio, gli ausiliari ir e venir ammettono la stessa morfologia verbale delle loro due controparti italiane andare e venire: all'interno delle perifrasi gerundivali i quattro verbi possono essere utilizzati sia in forma semplice sia in forma composta (Moretti / Orvieto 1979: 201). Ciononostante, è evidente che le due perifrasi italiane andare + gerundio e venire + gerundio sono molto scarsamente utilizzate nella lingua d'uso comune ${ }^{12}$, cosa che non può dirsi delle

10 Per ulteriori approfondimenti sulle altre, numerose valenze aspettuali che può assumere la perifrasi venir + gerundio, vedasi anche Ambrosini (2008: 116-122).

Per ulteriori approfondimenti, vedasi anche Bertinetto (1997).

12 Notiamo, però, che non mancano diverse distribuzioni d'uso diatopiche di andare e venire + gerundio. Se è vero che nell'italiano standard o neo-standard esse non compaiono, in alcune varietà regionali d'italiano il loro uso nel parlato quotidiano è più frequente. È, ad esempio, il caso dell'italiano parlato in Sardegna: in tale varietà, il gerundio è più produttivo che nello standard, ed è più incline a esprimere valori aspettuali combinandosi in perifrasi non solo con gli ausiliari andare e venire, ma anche con essere (Caddeo 2019; Olesen 2010). In questo lavoro non possiamo occuparci approfonditamente di come la variazione diatopica dell'italiano possa essere efficacemente messa in relazione alla didattica delle tre perifrasi in oggetto. Rimandiamo, tuttavia, a Lavinio 
corrispondenti spagnole, che compaiono trasversalmente in tutte le varietà del sistema. Le cause di questa divergenza vanno ricercate nella valenza strumentale più o meno sviluppata dagli ausiliari andare e venire durante il loro processo di grammaticalizzazione, valenza strumentale che si riflette direttamente nelle categorie azionali ammesse dalle perifrasi in cui intervengono i due ausiliari. In una perifrasi molto grammaticalizzata, infatti, a un maggior valore strumentale dell'ausiliare si accompagnerà un'incidenza via via minore della componente azionale del verbo principale, come nel caso degli ausiliari stare e estar. Di conseguenza, si ridurranno progressivamente le restrizioni azionali che limitano l'uso della perifrasi, per cui essa espleterà una gamma di valenze strumentali sempre più ampia, diffondendosi e consolidandosi nell' uso da parte dei nativi. Non è però questo il caso di andare + gerundio e venire + gerundio, che sono perifrasi solo parzialmente grammaticalizzate: il valore deittico-spaziale originale dei due ausiliari si è solo parzialmente riconvertito in un valore aspettuale telico-durativo ${ }^{13}$, come osserva Giacalone Ramat (1995: 200):

andare + gerundio, e venire + gerundio in modo ancora più marcato, sarebbero esempi di grammaticalizzazione interrotta, non solo per il calo della loro frequenza, ma anche per la loro specializzazione e idiomatizzazione. Essa si manifesta con la restrizione delle classi di aspetto lessicale dopo andare e venire e con certe restrizioni morfologiche e lessicali su venire, che privilegia i tempi perfettivi e tende a fossilizzarsi in determinate forme, quali andare dicendo.

Le restrizioni a cui fa riferimento Giacalone Ramat sono particolarmente importanti per venire + gerundio: essendo rimasta ancora fortemente vincolata al significato di spostamento fisico dell'ausiliare, su questa perifrasi gravano restrizioni lessicali più consistenti di quelle di andare + gerundio. Ne consegue, per esempio, la sua incompatibilità con i verbi di stato: venir essendo, ad esempio, è grammaticale, mentre la controparte spagnola venir siendo è una forma corretta e frequente nello spagnolo attuale (Bertinetto 1991:141; Barbagallo 2014:139).

Ir + gerundio, dal canto suo, ha sostanzialmente le stesse restrizioni azionali del modello perifrastico italiano corrispondente; tuttavia, il grado di grammaticalizzazione di ir lo ha portato a sviluppare una gamma di valori strumentali più ampia rispetto ad andare, il che spiega perché la presenza della perifrasi spagnola abbia prosperato ben di più della corrispondente italiana (Sanchez Montero 1993: 104).

A tal proposito, sono molto significativi i dati raccolti nel corpus di Brianti (2000), che è composto da una serie di testi di prosa letteraria e giornalistica italiana di due periodi che vanno, rispettivamente, dal 1800 al 1847 e dal 1985 al 1988: nel primo periodo analizzato, la perifrasi andare + gerundio supera stare + gerundio del $130 \%$ nei testi giornalistici, e del $200 \%$ in quelli letterari. Nel secondo periodo, stare + gerundio è superiore ad andare + gerundio dell' $1,106 \%$ nei testi giornalistici e del $463 \%$ nella prosa letteraria. La presenza della perifrasi venire + gerundio appare già molto ridotta all'inizio dell'analisi di Brianti. Secondo Brianti (2000: 107), quest'ul-

(2018) per recenti approfondimenti sull'utilità della riflessione varietistica, anche diatopica, dell'italiano; tale utilità, riteniamo, può essere estesa anche ai contesti di insegnamento di L2, a patto che avvenga a livelli di competenza avanzati, in cui siano già sedimentate buone basi comunicative in varietà standard della nostra lingua.

13 Per ulteriori approfondimenti in termini di aspetto e azionalità che tipizzano andare + gerundio e venire + gerundio, vedasi Bertinetto (1991: 138-147; 1997: 160-180). 
tima perifrasi: «[...] è di uso molto più limitato rispetto a quella con andare, e la sua frequenza, invece che aumentare, continua a calare nel corso dei secoli (eccetto un lieve rialzo nel '700). Ad esempio, venire + gerundio è 5 volte meno impiegata di andare + gerundio nei testi del '300, 12 volte nel ' 500 e ben 33 volte nel '700»).

Queste premesse spiegano come, nella lingua italiana attuale, le perifrasi andare + gerundio e venire + gerundio siano ormai utilizzate molto raramente e, cosa ancora più interessante, solo in alcune varietà di repertorio: nelle comunicazioni di registro medio-alto o alto, a canale prevalentemente scritto, fruibili solo da parte di parlanti con un certo livello d'istruzione. Tali varietà sono poco in contatto con la lingua di uso medio che si aspira a proporre e insegnare in sede didattica, quanto meno nei livelli basici e intermedi.

Nello spagnolo attuale, invece, l'uso di ir + gerundio e venir + gerundio è corrente $^{14}$, e non solamente in comunicazioni di registro elevato: al contrario, queste perifrasi ricorrono nella parlata quotidiana dei nativi, anche in contesti di registro colloquiale-informale, o persino gergale.

Per questa ragione, al di là della piena corrispondenza formale tra il paradigma delle due perifrasi spagnole e quello delle due perifrasi italiane, è evidente che la loro non piena corrispondenza a livello funzionale dipende non tanto da un criterio grammaticale, quanto da norme sociolinguistiche. Detto in altri termini:

A. vi sarà piena corrispondenza funzionale tra $i r+$ gerundio/venir + gerundio e andare + gerundio/venire + gerundio solo se il contesto comunicativo dato si inserirà in un livello diafasico in cui risulta adeguato l'uso di queste perifrasi sia in LM sia in LO; in buona sostanza, vi potrà essere piena corrispondenza funzionale solamente se la comunicazione in oggetto sarà di registro medio-alto o alto, come nei seguenti esempi:

\section{Tabella 1}

\begin{tabular}{|c|c|c|}
\hline REGISTRO & SPAGNOLO & ITALIANO \\
\hline alto, lingua letteraria & $\begin{array}{l}\text { 21) Poco después Flimnap se en- } \\
\text { contró con un grupo de noticie- } \\
\text { ros de los grandes diarios, que le } \\
\text { iban buscando desde horas antes } \\
\text { (Blasco Ibáñez 2008: } 163 \text { ) }\end{array}$ & $\begin{array}{l}\text { Poco dopo, Flimnap si imbatté } \\
\text { in un gruppo di giornalisti di } \\
\text { quotidiani di spicco, che lo an- } \\
\text { davano cercando da ore. }\end{array}$ \\
\hline $\begin{array}{l}\text { medio-alto, lingua } \\
\text { del giornalismo }\end{array}$ & $\begin{array}{l}\text { 22) Las fuerzas militares rusas } \\
\text { consiguieron entrar tras haber } \\
\text { introducido en el recinto una } \\
\text { mezcla de sustancias deriva- } \\
\text { das del fentanilo [...]. Pero de- } \\
\text { sde décadas anteriores se viene } \\
\text { experimentando en estos términ- } \\
\text { os. (S. Font 2009) }\end{array}$ & $\begin{array}{l}\text { Le forze militari russe riusciro- } \\
\text { no ad entrare dopo aver intro- } \\
\text { dotto nel recinto una miscela } \\
\text { di sostanze derivate dal fentanil } \\
{[\ldots] \text {. Ma già da decenni si viene }} \\
\text { sperimentando in questi termi- } \\
\text { ni. }\end{array}$ \\
\hline
\end{tabular}

14 Sulla differenza in termini di frequenza d'uso tra andare/venire + gerundio e ir/venir + gerundio si veda, ad esempio, Martínez Atienza (2017: 31). 
I casi riassunti in tabella 1 sono transfer dall'esito grammaticale e funzionale positivo, in quanto le perifrasi spagnole di partenza trovano un pieno corrispettivo, anche varietistico, in italiano.

B. La corrispondenza formale tra le due coppie di perifrasi viene a mancare nel momento in cui la situazione comunicativa sarà di registro medio o inferiore al medio: in questi casi, contrariamente a quanto accade in spagnolo, l'uso di andare + gerundio o venire + gerundio, seppur non contestabile sul piano prettamente grammaticale, risulta piuttosto inadeguato sul piano diafasico, come si evince dai seguenti esempi:

\section{Tabella 2}

\begin{tabular}{|c|c|c|}
\hline REGISTRO & SPAGNOLO & ITALIANO \\
\hline lingua di uso medio & $\begin{array}{l}\text { 23) Ha ido perdiendo peso/adel- } \\
\text { gazando desde que empezó el } \\
\text { tratamiento (García Fernández } \\
\text { 2006: 174) }\end{array}$ & $\begin{array}{l}\text { Da quando è iniziato il tratta- } \\
\text { mento, è andato perdendo peso/ } \\
\text { dimagrendo (?) }\end{array}$ \\
\hline lingua di uso medio & $\begin{array}{l}\text { 24) Viene contando las mismas } \\
\text { cosas desde hace mucho tiempo } \\
\text { (Trovato 2014: 49). }\end{array}$ & $\begin{array}{l}\text { È da tempo che viene raccontan- } \\
\text { do (?) la stessa storia }\end{array}$ \\
\hline $\begin{array}{l}\text { lingua colloquia- } \\
\text { le-informale }\end{array}$ & $\begin{array}{l}\text { 25) Brad Pitt parece seguir inten- } \\
\text { tando conseguir la custodia com- } \\
\text { partida de sus hijos [...]. A lo lar- } \\
\text { go de estos últimos años el actor } \\
\text { se ha ido cabreando más debido } \\
\text { a esta circunstancia (LaCosaRo- } \\
\text { sa.com 2016) }\end{array}$ & $\begin{array}{l}\text { Sembra che Brad Pitt continui a } \\
\text { lottare per ottenere l'affidamento } \\
\text { congiunto dei suoi figli [...]. Ne- } \\
\text { gli ultimi anni, l'attore si è an- } \\
\text { dato scocciando/irritando (?) a } \\
\text { causa di questa circostanza. }\end{array}$ \\
\hline $\begin{array}{l}\text { registro volga- } \\
\text { re-substandard }\end{array}$ & $\begin{array}{l}\text { 26) Los árbitros nos han jodi- } \\
\text { do o nos vienen jodiendo desde } \\
\text { hace ya varios años, pero aun así } \\
\text { se puede ganar ligas (foros.abc. } \\
\text { com 2015) }\end{array}$ & $\begin{array}{l}\text { Gli arbitri ci hanno fregati o ci } \\
\text { sono venuti fregando (?) per } \\
\text { anni, ma si possono ancora vin- } \\
\text { cere campionati. }\end{array}$ \\
\hline
\end{tabular}

Questi casi, a differenza di quelli illustrati nella Tabella 1, possono considerarsi realizzazioni di un transfer di addizione che aggiunge, in un contesto varietistico errato, due strutture perifrastiche inadeguate, in netto contrasto con il tenore comunicativo.

C. Non dimentichiamo, infine, il caso - cui si accennava in precedenza - in cui venir + gerundio si combina con un verbo di stato, come ad esempio ser, come in:

27) Hace años que Colombia viene siendo un equipo muy competitivo. (Cárdenas Utrilla 2020) 
Una realizzazione quale *viene essendo come corrispondente della spagnola viene siendo rappresenterebbe un esempio di transfer per addizione dall'esito negativo: un apprendente che abbinasse all'ausiliare venire un verbo ausiliato stativo - ignorando le restrizioni azionali previste per la lingua bersaglio - produrrebbe dunque una struttura agrammaticale in italiano.

\subsection{Dati sul transfer delle perifrasi}

Uno dei più evidenti punti di divergenza fra italiano e spagnolo risiede nella maggiore frequenza e produttività dei costrutti analitici di quest'ultima lingua. Se l'impianto verbale sintetico dei due idiomi è facilmente sovrapponibile ${ }^{15}$, è proprio la presenza di un ampio repertorio di costrutti perifrastici tempo/modali a segnare una sostanziale differenza: in spagnolo si annoverano, infatti, circa 59 perifrasi di uso frequente (García Fernández / Krivochen 2019: 57-58) ${ }^{16}$, mentre in italiano le controparti sono molte meno, e spesso il loro uso è marcato in determinate varietà d'uso (Barbagallo 2014; Musto / Ripa 2005).

Cercheremo ora traccia della manifestazione di questo "sbilanciamento" analitico dello spagnolo nelle interlingue di hispanohablantes apprendenti di italiano. Verificheremo da diverse fonti se è attestato il transfer superfluo delle tre perifrasi oggetto di indagine di questo lavoro e, laddove possibile, verificheremo se questo fenomeno permane anche a livelli di competenza più avanzati.

Il libro di Bailini (2016) fornisce un quadro analitico delle caratteristiche delle interlingue degli ispanofoni apprendenti di italiano, e lo fa sulla base dell'analisi del corpus CORITE, costituito da 358 testi scritti prodotti da apprendenti di livelli di competenza fra l'A1 e il B1 (Bailini / Frigerio 2018). Sebbene si tratti di dati scritti, dunque non completamente spontanei perché suscettibili a controllo metalinguistico da parte dello scrivente, va notato che essi provengono da interazioni informali via tandem o e-mail, certamente meno sorvegliate di altre tipologie di scritture. Inoltre, CORITE ha carattere longitudinale e permette di verificare gli eventuali mutamenti interlinguistici degli scriventi nel corso dei mesi. Prendiamo infine anche in considerazione il fatto che rintracciare un fenomeno di transfer nelle produzioni scritte è segno di ulteriore fossilizzazione e di difficoltà a distaccarsi dal modello della LM anche in un contesto comunicativo in cui vi è la possibilità di controllare adeguatamente le proprie produzioni. In prima istanza, Bailini (2016, cap. 3) passa in rassegna 48 studi sulle caratteristiche dell'italiano degli ispanofoni. In questi studi è rilevata la mappatura su stare + gerundio del valore aspettuale continuativo, che ingenera il suo abbinamento con

15 Non possiamo qui dilungarci oltre sugli esiti del contatto fra spagnolo e italiano, esiti che differiscono, per altro, anche sulla base delle situazioni sociolinguistiche che contribuiscono a determinarli. Per quanto riguarda la morfologia e la trasferibilità di morfemi legati dall'una all'altra lingua, rimandiamo il lettore a Schmid (2015) e alle interessanti considerazioni sui vincoli di trasferibilità dovuti a gradienti di naturalezza e marcatezza morfologica.

16 Anche l'inventario proposto dalla Gramática descriptiva de la lengua española annovera un totale di 59 perifrasi. Più esattamente, Yllera (1999: 3323-3388) identifica 15 perifrasis de gerundio e 15 perifrasis de participio, e Gómez Torrego (1999: 3392-3441) identifica 29 perifrasis de infinitivo. Tuttavia, come spiegano gli stessi autori, questi conteggi possono variare anche sensibilmente in base al fatto che la stessa definizione del termine perifrasi e delle strutture ad essa riconducibili discenda da criteri di ordine sintattico o piuttosto da altri di ordine semantico. In tal senso, al momento, non è possibile stabilire una nomenclatura unica e assoluta per tutte le perifrasi esistenti in lingua spagnola, questione ricorrente nella bibliografia di riferimento dedicata alla loro descrizione (Olbertz 1998; Fernández de Castro 2000; RAE-ASALE 2009). 
tempi composti, dando origine a strutture come * sono stato lavorando tutto il giorno. È inoltre rilevata la presenza superflua di strutture perifrastiche costituite da verbi di movimento + gerundio, fra cui andare + gerundio (Bailini 2016: 99). Trasferimenti superflui di perifrasi verbali dalla LM alla LO si rinvengono anche nell'analisi precipua del corpus CORITE, presentata nella seconda parte dell'opera di Bailini (2016: 216-218): vengono riportati casi di usi impropri di stare + gerundio e di andare/venire + gerundio fino al livello B1, il più alto considerato in CORITE.

Spostandoci ora verso il filone di indagine della sociolinguistica del contatto, prendiamo in analisi due ricerche che basano le loro indagini su corpora orali, composti da registrazioni di parlato spontaneo e semispontaneo di ispanofoni apprendenti non istruiti di italiano. Partecipano alla ricerca di Schmid (1994) 12 ispanofoni residenti a Zurigo da una media di 14 anni. L'obiettivo del ricercatore è verificare le strategie d'acquisizione e le caratteristiche delle interlingue nate dal contatto fra lo spagnolo e il Fremdarbeiteritalienish, la varietà d'italiano usata come lingua franca da molti immigrati romanzofoni nella Svizzera tedesca, appresa spesso molto meglio delle varietà locali di tedesco o del Hochdeutsch. Il ricorso a strutture perifrastiche tipiche della LM ma assenti nella LO è ben attestato da Schmid, che riporta l'ampio uso di andare e venire + gerundio in contesti informali, a cui gli informanti ricorrono nel tentativo di esprimere valori aspettuali progressivi (Schmid 1994: 195-196).

La ricerca di Vietti (2005) si muove sempre nello stesso paradigma linguistico e "arruola" come informanti 15 donne peruviane immigrate a Torino, città in cui risiedono da una media di 5,8 anni. Le interviste semistrutturate a cui le quindici signore hanno partecipato forniscono dati molto interessanti sul legame fra livello di integrazione e caratteristiche delle interlingue, in particolar modo in merito alla correttezza d'uso di parole funzionali (come alcune preposizioni) notoriamente complesse per gli ispanofoni. Il corpus che ne emerge è molto ricco ed è composto da interlingue intermedio/avanzate di parlanti anche discretamente competenti. Non mancano però, anche qui, rilievi di transfer superfluo verso l'italiano delle nostre tre perifrasi: in più punti (Vietti 2005: 18; 121) l'autore riporta la presenza di costruzioni perifrastiche progressive costituite da andare/venire + gerundio, ed è pure rilevata la mappatura dell'aspetto continuativo su stare + gerundio, con esiti agrammaticali di combinazione con tempi passati perfettivi (cfr. anche Vietti 2010: 226 per ulteriori dati).

Di quest'ultima perifrasi, ma in chiave psicolinguistica e sperimentale, si è occupato lo studio di Della Putta e Strik Lievers (2020), che ha verificato la capacità di non trasferire l'intera portata aspettuale di estar + gerundio alla controparte italiana stare + gerundio. 108 ispanofoni studenti di italiano hanno preso parte allo studio. Basandosi sui risultati di un test CILS di livello B1, gli autori hanno suddiviso gli informanti in tre livelli di competenza: principianti, intermedi e avanzati, a loro volta classificati sulla base del contesto di studio (L2 vs. LS) dell'italiano. I partecipanti sono stati sottoposti a un task di giudizi di grammaticalità temporizzati in cui comparivano usi di stare + gerundio che ricalcavano il modello dello spagnolo; subito dopo, è stato somministrato un immediate recall test per verificare quali fossero gli errori effettivamente giudicati dagli studenti ${ }^{17}$, e i dati dei giudizi di grammaticalità

\footnotetext{
È stato così notato che in molti casi il giudizio di agrammaticalità di frasi come * sono stato ballando tutta la sera non dipendeva dall'uso di stare + gerundio con tempi composti, ma dall'ausiliare essere che, nella rappresentazione degli apprendenti, sostituiva erroneamente l'ausiliare ritenuto corretto, ovvero avere, su pieno modello dello spagnolo.
} 
sono stati ricalcolati sulla base delle risposte elicitate da questo ultimo compito. I risultati rivelano che anche ai livelli più avanzati rimane una grossa incertezza in merito all'agrammaticalità di stare + gerundio abbinata a tempi composti e a valori aspettuali continuativi: più del $50 \%$ delle risposte degli apprendenti avanzati è infatti errata, e dagli immediate recall test non traspare una ferma consapevolezza dei limiti da imporre, in italiano, alla perifrasi spagnola estar + gerundio.

Ambrosini (2018) ha sottoposto a un test di completamento testuale e frasale non temporizzato 78 studenti ispanofoni di italiano iscritti presso due Escuelas de Idiomas (EOI) in Spagna, una a Madrid e una a Barcellona ${ }^{18}$. Tutti i partecipanti allo studio hanno un livello di competenza intermedio o alto (di media un B2 del QCER) e sono in generale parlanti molto competenti di italiano. È molto interessante notare come anche in questo studio gli apprendenti accettino per il 50\% - dato della EOI di Madrid - e per il 71\% - dato della EOI di Barcellona - dei casi la frase sono stato studiando; lo stesso studio riporta che la perifrasi ero stato dormendo è accettata dal $47 \%$ degli informanti del primo dei due gruppi, e dal 34\% degli informanti del secondo. Tale linea di tendenza è affine a quanto rilevato in Della Putta e Strik Lievers (2020), dove, come riportato poco sopra, la correttezza nei giudizi di grammaticalità di questo tipo di frasi non superava il $50 \%$ per gli informanti con competenze avanzate. Il lavoro di Ambrosini restituisce, altresì, grosse difficoltà degli ispanofoni nel non usare le perifrasi andare/venire + gerundio in contesti diafasici italiani standard o sub standard, rilevando, al contrario, un'ampia tendenza da parte degli informanti a utilizzare le due perifrasi in contesti comunicativi simili a quelli proposti a titolo d'esempio nella tabella 2.

$\mathrm{Su}$ questa stessa linea, possiamo segnalare anche i dati raccolti in Ambrosini (2020). Lo studio presenta una parte di raccolta dati realizzata tramite la somministrazione di un test non temporizzato ad apprendenti di italiano ispanofoni di tre Escuelas de Idiomas dell'area di Madrid ${ }^{19}$, in corsi di livello B1, B2 e C1. Nel test si proponeva agli informanti un'intervista scritta in italiano contenente quattro forme calcate di stare + gerundio (ero stato vivendo, sono stato lavorando, ero stato studiando, sono stato festeggiando), sulle quali gli informanti erano chiamati ad esprimere un giudizio di grammaticalità. I risultati del test confermano che un'ampia percentuale di informanti, in tutte e tre le EOI coinvolte e in tutti e tre i livelli di competenza considerati, ritengono grammaticali queste forme; si pensi che la perifrasi sono stato lavorando, ad esempio, riporta un giudizio di accettabilità che tocca una percentuale massima del $93 \%$ in uno dei tre gruppi di informanti di livello B1 per "ridursi" solamente fino a una percentuale minima del 38,5\% in uno dei tre gruppi di livello C1.

Questa breve rassegna di studi, a cui vanno aggiunte "tracce" dell'uso improprio di queste perifrasi anche in altre ricerche (cfr., inter alia, Morgana / Zaffaroni 2011: 199), testimonia la difficoltà dell'inibizione dell'uso di queste tre strutture. Accanto ai problemi di analisi di input esposti in $\$ 2$, una lettura di ispirazione costruzionista e cognitivista di queste difficoltà acquisizionali può essere d'ulteriore supporto alla nostra argomentazione. Secondo gli approcci costruzionisti alla grammatica à la

18 Partecipano allo studio studenti di italiano della Escuela Oficial de Idiomas Drassanes di Barcellona e della Escuela Oficial de Idiomas Jesús Mestro di Madrid. Nel primo caso, Ambrosini ha raccolto separatamente i risultati degli informanti esclusivamente ispanofoni e quelli degli apprendenti bilingui calalano-ispanofoni. Tralasciamo qui i risultati degli informanti catalanofoni, per questioni prospettiche.

19 Scuole che hanno partecipato allo studio: EOI Jesús Maestro, EOI Alcorcón, EOI Alcalá de Henares. 
Goldberg $(2003)^{20}$, le perifrasi assumono lo status di costruzioni parzialmente costituite (partially filled-in constructions), in cui due elementi sono fissi (il verbo di stato o di movimento e l'eventuale preposizione), mentre il verbo alla forma non finita è il costituente semanticamente variabile (cfr. Goldberg 2003: 219). Secondo la prospettiva costruzionista, le perifrasi sono dunque pattern linguistici il cui significato non è inferibile dalla semplice "somma semantica" dei loro costituenti: il meccanismo cognitivo che spiega l'assunzione di valori tempo-aspettuali di questi costrutti è la metafora concettuale TEMPO È SPAZIO, che permette lo shift cognitivo del verbo finito dal dominio motorio a quello tempo-aspettuale assunto all'interno della costruzione perifrastica. L'ampissima diffusione fra le lingue del mondo di questa metafora porterebbe gli apprendenti a trasferire con più facilità dalla LM alla LO il processo metaforico soggiacente alle costruzioni perifrastiche, estendendo all'italiano lo scarto semantico del verbo finito spagnolo; secondo Odlin, infatti, quando i domini metaforici sono ritenuti coincidenti dagli apprendenti, «they assume certain constructions to be universal when in fact they involve language-specific meaning extension» (Odlin 2008: 325). Occorre dunque approcciare l'erroneo transfer di addizione delle perifrasi da un punto di vista didattico, cercando soluzioni adatte ad aiutare gli ispanofoni a venire a capo di questo problema di transfer, particolarmente resistente sia all'istruzione formale sia all'esposizione spontanea ad abbondante input.

\section{Presenza e trattazione delle tre perifrasi nelle grammatiche di lingua italiana}

Consideriamo ora opportuno proporre un'analisi di come sono trattate le perifrasi stare e andare/venire + gerundio nei più comuni supporti nozionali attualmente disponibili per gli apprendenti ispanofoni di italiano, focalizzandoci in particolare sulla presenza o sull'assenza di riflessioni contrastive che possano indicare i limiti d'uso da imporre alle controparti spagnole. Per supporti nozionali ci riferiamo in prima istanza ai materiali didattici - in particolar modo alle grammatiche di lingua italiana - attraverso i quali gli apprendenti possono accostarsi a queste strutture linguistiche e alle loro norme di funzionamento in LO. Il ruolo di questi materiali didattici è essenziale in quanto costituiscono, se debitamente integrati dall'accompagnamento del docente, un utile strumento in grado di influenzare la velocità e l'efficacia con cui l'apprendente si smarcherà dalle forme e regole della LM per approdare a quelle della LO.

Nel caso delle perifrasi qui considerate, ci è sembrato interessante rilevare una differenza significativa tra la presenza e la modalità di trattazione di stare + gerundio, da un lato, e la pressoché totale assenza di trattazione di andare + gerundio e venire + gerundio, dall'altro; differenza che, probabilmente, genera e riflette al tempo stesso le distinte modalità di transfer che abbiamo esemplificato in $\S 3.1$ e $\S 3.2$, e che

La Linguistica cognitiva è costituita da diversi modelli teorici che condividono l'obiettivo di spiegare il funzionamento del linguaggio considerandolo un'abilità cognitiva integrata, dunque non scindibile dalla nostra esperienza fisica, mentale e culturale. In particolare, secondo alcuni paradigmi della Linguistica cognitiva (come la Grammatica delle Costruzioni o la Grammatica Cognitiva, cfr. Masini 2016 e Langacker 1988), una lingua sarebbe costituita essenzialmente da costruzioni simboliche non-arbitrarie, ovvero da associazioni di forme e significati la cui esistenza è motivata da meccanismi cognitivi generali di natura percettiva e categorizzativa (come le metafore concettuali) che sono, in ultima analisi, costituiti e modellati dall'esperienza corporea e sociale dell'individuo. 
ci offre altresì una concreta esemplificazione di quelle che in $\S 2$ abbiamo definito come prove positive e prove negative indirette nella LO.

Per poter osservare in che modo le perifrasi in oggetto sono (o non sono) tematizzate nelle attuali grammatiche di lingua italiana per stranieri, abbiamo consultato $i$ dati raccolti in Ambrosini (2018: 216-223) e Oricco (2020: 24-48). Nel primo dei due studi, sono state considerate dodici grammatiche di lingua italiana per stranieri; di queste, undici sono grammatiche rivolte a un pubblico di apprendenti internazionale (non specifico), mentre una è rivolta a un pubblico di apprendenti ispanofo$\mathrm{ni}^{21}$; nel secondo studio, invece, l'analisi dei materiali ha avuto come oggetto cinque grammatiche di lingua italiana rivolte a un pubblico di apprendenti ispanofoni in contesto universitario ${ }^{22}$.

Consideriamo per prima la perifrasi stare + gerundio: essa è presente in tutte le pubblicazioni consultate nei due studi, nei quali si presentano sia la sua struttura morfologica, sia le norme che regolano il suo uso nella lingua italiana attuale. Inoltre, si offrono esempi di un suo uso in contesto, e si propongono allo studente esercizi applicativi perché ne faccia pratica e la interiorizzi progressivamente. La trattazione della perifrasi stare + gerundio è giustificata dal fatto che essa è la perifrasi aspettuale probabilmente più diffusa, nell'italiano attuale, pertanto il suo studio viene tendenzialmente proposto nelle programmazioni didattiche già a partire da un livello $\mathrm{A} 2^{23}$. La scelta di introdurre questa struttura sin da questo livello di competenza risulta ancor più idonea se si considera una dinamica di affinità linguistica tra LM e LO come quella che contraddistingue, per l'appunto, spagnolo e italiano.

Ciò che manca nella trattazione proposta in queste grammatiche, al di là della trattazione dell'innegabile corrispondenza formale tra estar + gerundio e stare + gerundio, è una riflessione sulla divergenza che abbiamo illustrato in \$3.1: non si spiega infatti che estar + gerundio è una perifrasi progressiva e durativa (che dunque si utilizza con verbi sia perfettivi che imperfettivi), mentre stare + gerundio può solo denotare un'azione in svolgimento e si utilizza solo con verbi imperfettivi. Si tratta di una riflessione latitante anche nel Manual de gramática italiana di Díaz, l'unico tra $i$ testi consultati ad essere stato pensato per un pubblico di apprendenti ispanofoni.

In Oricco (2020) si documenta una tendenza del tutto analoga: su cinque grammatiche di lingua italiana per apprendenti ispanofoni, che presumibilmente potrebbero offrire una riflessione più calibrata sia sulle affinità che sulle divergenze nel rapporto tra la LM e la LO coinvolte, solamente una ${ }^{24}$ osserva che stare + gerundio,

$\overline{21}$ Il principale criterio di selezione delle grammatiche è stato cronologico: la meno recente è quella di Patota, Grammatica di riferimento dell'italiano contemporaneo, pubblicata da garzanti nel 2006. La grammatica rivolta al pubblico ispanofono è ad opera di Carrera Díaz (2015, edizione originale 1985), Manual de gramática italiana.

22 Specifica Oricco (2020: 22): «La ricerca si è svolta esclusivamente in ambito universitario. Sono stati controllati i programmi di vari corsi di italiano offerti in diverse università spagnole, per appurare quali libri fossero stati consigliati (in particolare: Malaga, Salamanca, Madrid, Oviedo, Barcellona, Zaragoza, Castilla La-Mancha, Extremadura). È necessario precisare che, nella maggior parte dei casi, i libri principali consigliati sono libri di testo generalisti quali Nuovo Contatto, Nuovo Espresso, ecc. Questi libri sono scritti in italiano, e possono essere utilizzati da qualsiasi apprendente di italiano, non sono quindi rivolti espressamente a un pubblico ispanofono; in questi manuali manca perciò l'analisi contrastiva tra l'italiano e lo spagnolo. In alcuni casi, tuttavia, soprattutto all'università di Oviedo, le grammatiche rivolte esclusivamente a ispanofoni sono obbligatorie. Com'è stato accennato nell'Introduzione di questo elaborato, le grammatiche scelte per la ricerca sono tutte grammatiche di lingue italiane destinate a ispanofoni».

23 Si vedano, ad esempio, i sillabi di riferimento per l'insegnamento dell'italiano lingua straniera di Benucci (2007) e Lo Duca (2006).

24 Si tratta del Curso de lengua italiana. Parte teorica di Carrera Díaz (1992), che spiega: «El peculiar funcionamiento de la secuencia STARE + GERUNDIO requiere, sin embargo, algunas matizaciones ulteriores: a) Dado 
perifrasi eminentemente imperfettiva in italiano, non ammette che l'ausiliare compaia in una forma composta. A simili conclusioni giunge la ricerca di Daloiso (2018), che si è precipuamente occupata della presenza della perifrasi stare + gerundio in sei recenti grammatiche di italiano per stranieri, non pensate solo per un pubblico ispanofono. Nella sua analisi, Daloiso (2018: 6) considera la presenza di una prospettiva comparativa «con altre lingue, anche in forma implicita (ad esempio, esplicitando alcune restrizioni d'uso della perifrasi progressiva che la differenziano da altre lingue molto diffuse, come l'inglese o lo spagnolo)». Tali considerazioni comparative sono rinvenute solo in due grammatiche, con modalità e portate esplicative che lo stesso Daloiso definisce come limitate.

In tutte le grammatiche pedagogiche considerate negli studi qui riassunti, le prove positive che concernono stare + gerundio sono variamente trattate: si esplicitano e si esercitano, cioè, le sue forme, i suoi contesti di apparizione e le funzioni che su di essa vengono mappate. Tuttavia, mancano quasi del tutto delle considerazioni sulle prove negative indirette di stare + gerundio, ovverosia su quali siano le forme e le funzioni che essa non può assumere in italiano. Questa mancanza può essere dunque molto svantaggiosa per quegli apprendenti che nella loro LM hanno strutture molto simili con portate d'uso più ampie di quelle dell'italiano: le grammatiche a cui fanno affidamento non li avvertono di quando non usare stare + gerundio, fallendo nel contraddire ipotesi interlinguistiche erronee.

Consideriamo ora le perifrasi andare + gerundio e venire + gerundio, analizzate nello studio di Ambrosini (2018). Solamente tre delle dodici grammatiche prese in analisi presentano una trattazione di queste due perifrasi; trattazione, peraltro, completamente assente anche nei sette manuali di lingua italiana per stranieri analizzati nella stessa ricerca. La tipizzazione che le tre grammatiche in questione riservano alle due perifrasi risulta comunque poco completa: se ne illustra in modo molto schematico la struttura morfologica e se ne offre qualche esempio di uso in contesto, senza però offrire all'apprendente alcuna informazione circa il profondo divario che separa le perifrasi italiane dalle spagnole in termini di adattabilità al contesto comunicativo dato e, conseguentemente, in termini di frequenza d'uso delle stesse nei rispettivi repertori linguistici.

Tirando le somme, pare che si possano evincere alcune tendenze generali sulla trattazione di queste tre perifrasi nelle grammatiche di lingua italiana attualmente in uso.

Nel caso di stare + gerundio, le grammatiche propongono un input nozionale esplicito sulla forma e sull'uso della perifrasi italiana, eminentemente progressiva, fornendone eventualmente alcuni esempi d'uso in contesto. Tali presupposti, tuttavia, quand'anche accompagnati da opportune e mirate indicazioni da parte del docente sui caratteri contrastivi che contraddistinguono il rapporto tra estar + gerundio e stare + gerundio, possono non costituire un deterrente sufficiente, dal momento che, come discusso in $\$ 3.1$, i calchi formali sull'uso di queste perifrasi sono piuttosto frequenti anche nell'interlingua di apprendenti di livello avanzato.

Per quanto riguarda andare + gerundio e venire + gerundio, del tutto esclusi sia

que en italiano el gerundio tiene un marcado carácter imperfectivo, no se puede utilizar con tiempo pasado, excepción hecha del imperfecto; se hace necesario, en caso, optar por una construcción personal o bien por la perífrasis de infinitivo: "He estado todo el día escuchando música": Versión 1: ho ascoltato musica tutto il giorno. Versión 2: Sono stato tutto il giorno ad ascoltare musica [...]» (p. 466). 
dall'indice di argomenti trattati in classe, sia in termini di riflessione metalinguistica mediata dal docente di italiano, l'apprendente ispanofono manca completamente di una norma di riferimento d'uso in LO. Si tratta di una situazione ovviamente comprensibile, in termini di programmazione didattica: nel momento in cui l'obbiettivo è quello di insegnare un italiano che sia quanto più possibile autentico, fruibile, vicino alla lingua media parlata dai nativi (Calvi 1995: 157), e vista anche la scarsità del monte ore previsto per la maggior parte dei percorsi didattici, difficilmente il docente potrà includere nella propria programmazione una parentesi sull'uso - ormai disuso - di andare + gerundio e venire + gerundio.

\section{Proposte pedagogiche}

Quest'ultimo paragrafo è dedicato all'estensione di alcune proposte didattiche pensate per lenire il problema del transfer superfluo delle tre perifrasi in oggetto. Includere in una progettazione didattica anche la trattazione di stare e andare/venire + gerundio appare essere necessario giacché, come visto in $\$ 2$ e $\$ 3.3$, le complessità psicolinguistiche di ristrutturazione di queste strutture da spagnolo a italiano sono difficilmente sormontabili. In primo luogo, abbiamo l'estrema opacità delle prove date dall'input che, nel caso della perifrasi progressiva, sono solo prove negative indirette; la situazione è ancora più complessa per andare/venire + gerundio: per accordare al modello della LO la loro portata significativa e i loro contesti d'uso, l'apprendente ispanofono deve riuscire a coordinare adeguatamente prove negative indirette (l'assenza di queste perifrasi in alcuni registri dell'italiano) e prove dirette (la presenza, seppur rara, delle due costruzioni in contesti diafasici particolari). A queste considerazioni aggiungiamo le naturali strategie d'apprendimento messe generalmente in campo da apprendenti di lingue affini, che poggiano le loro supposizioni e la loro attività comunicativa più sulla ricerca delle somiglianze che delle differenze (Ringbom 2007: cap. 1) e che tendono a fare paragoni strutturali ingenui fra le proprietà della LM e della LO. Infine, le considerazioni fatte in $\S 4$ sui materiali di studio dell'italiano, che con estrema rarità contemplano delle adeguate analisi delle restrizioni d'uso, in italiano standard, delle tre perifrasi in oggetto.

In quanto segue, quindi, proporremo delle attività e delle accortezze glottodidattiche a nostro parere utili per aiutare gli ispanofoni ad allineare adeguatamente le loro ipotesi interlinguistiche su stare e andare/venire + gerundio alle proprietà dell'italiano. La generalizzabilità pedagogica di quanto esporremo è certamente un limite non indifferente: come ben evidenziato da Spada (2019), è spesso difficile per gli insegnanti applicare adeguatamente alla loro prassi le indicazioni operative che vengono dalla ricerca. Ogni docente, infatti, esercita il suo "senso di plausibilità" (Ur 2019) in merito alle indicazioni pedagogiche che vengono dal mondo della ricerca, e così facendo discrimina fra attività possibili e utili e attività inadeguate a un determinato pubblico di studenti. Mantenendo saldo questo principio pragmatico e non necessariamente ottimale, anche facendo riferimento a lavori sperimentali passati raccogliamo in quanto segue alcune possibilità operative. 


\subsection{Considerare l'esistenza delle prove negative indirette}

Come abbiamo visto in $\S 2$, lo sviluppo interlinguistico basato su prove negative indirette è un processo assai complesso che deve essere guidato soprattutto in contesti di lingue simili, dove la tendenza è, appunto, quella di fare più affidamento alle somiglianze che alle differenze. In generale, la ricerca dice che quando l'interlingua si può ristrutturare solo o principalmente tramite prove negative indirette sono necessari interventi correttivi mirati da parte dell'insegnante, che in questo modo può aiutare il corretto allineamento dell'interlingua alle proprietà della LO (cfr. discussione in Della Putta 2018). Si delinea, quindi, un diverso indice di marcatezza pedagogica: le componenti sintetiche del sistema morfosintattico dell'italiano vengono acquisite con discreta facilità (date alcune condizioni sociologiche ed esperienziali facilitanti, cfr. Vietti 2010) dagli ispanofoni, mentre è proprio il ricorso a strutture analitiche spagnole che, per esprimere i medesimi valori aspettuali, hanno solo controparti sintetiche in italiano a richiedere particolare attenzione.

Occorre innanzitutto, quindi, dare consapevolezza agli insegnanti che le relazioni strutturali fra le due lingue possono creare delle "nicchie" d'ombra in cui le discrepanze si "nascondono" alla processazione dell'input compiuta dagli apprendenti. Come abbiamo visto, questa consapevolezza non sembra essere presente nelle grammatiche pedagogiche, che ignorano o non ritengono utile fare luce su questi ambiti di complessità. Un'adeguata attività comparativa fra i due codici, volta a rintracciare anche questo tipo di asimmetrie, appare essere necessaria sia in fase di pianificazione delle lezioni sia con gli studenti in classe. Non si tratta - ci teniamo a precisarlo - di una visione contrastiva tout-court: per essere apprese dagli ispanofoni, molte strutture dell'italiano non necessitano quasi di istruzione mirata e comparativa, dato che beneficiano semplicemente di abbondante esposizione all'input e di uso adeguato della LO. Le tre perifrasi in questione (e altre, cfr. Della Putta 2015), però, si prestano a un esercizio contrastivo mirato. Ciò che un insegnante può (e forse deve) fare, in questi contesti, è scoraggiare l'eccessivo ricorso alla strategia della congruenza, simulando incomprensione quando l'apprendente ispanofono usa erroneamente una delle tre strutture in oggetto. Creare un'incomprensione, ancorché simulata, può indurre l'apprendente a rendersi conto che nella sua enunciazione vi è un problema, e che tale problema va analizzato e riparato. Nelle parole di Long (1996, cit. in Valentini 2016: 13):

Paradossalmente, l'input comprensibile può inibire l'acquisizione. La mancata comprensione può talora essere necessaria per l'avanzamento dell'acquisizione stessa. Impasses comunicativi possono indurre l'apprendente a rendersi conto che esiste un problema linguistico, portandolo a prestare attenzione alla forma, a identificare il problema e a individuare nell'input stesso la nuova forma necessaria per superarlo.

I modi per attuare questa incomprensione sono parecchi: in Della Putta 2015 si presentano alcuni esempi in cui l'insegnante "gioca" sul mancato shift metaforico spazio/tempo/aspetto di alcuni verbi di movimento italiani come andare e tornare, che spesso vengono erroneamente usati dagli ispanofoni in costrutti perifrastici tipici della loro LM come andare e tornare a + infinito. Nei casi da noi contemplati, gli insegnanti potranno facilmente indicare gli errori d'uso diafasico di andare e venire 
+ gerundio chiedendo in maniera ironica, ad esempio, in quale epoca lo studente viva e a quale epoca risalga l'italiano che sta parlando. Questo bloccherà il flusso comunicativo lasciando in un qualche modo stupito il discente ispanofono, che ricorre a tali strutture perifrastiche con estrema disinvoltura. Una conseguente spiegazione più o meno esplicita, a seconda della sensibilità dell'insegnante e della classe, può favorire il consciousness raising verso le limitazioni d'uso, in italiano, di tali strutture.

A queste attività dialogiche possono essere fatte seguire, ciclicamente, correzioni mirate quando necessario o brevi focus on forms sulle divergenze perifrastiche fra i due codici. Utile è anche favorire l'uso di andare/venire + gerundio in contesti in cui realmente, in italiano, le possiamo incontrare: attività coinvolgenti come semplici role play fra studenti in cui si inscenano ruoli diacronicamente e diafasicamente marcati sono utili per dare il corretto spazio sia a prove negative (dirette e indirette) sia a prove positive che indicano quando si possono usare tali strutture anche nella LO.

\subsection{Manipolazione comparativa dell'input}

Un secondo tipo di attività che possono giovare alla corretta ristrutturazione interlinguistica riguardano il potenziamento dell' input scritto che, se adeguatamente manipolato, può fornire con maggiore efficacia quegli indizi contestuali utili per notare l'assenza, in (alcuni registri dell') italiano, delle tre perifrasi in oggetto. Potenziare l'input significa favorire la possibilità, tramite manipolazione grafica, di rendersi conto (i.e. noticing, cfr. discussione in Valentini 2016) della presenza o della co-occorrenza di elementi linguistici che favoriscono l'attivazione di determinate strutture o, nel nostro caso, che sarebbero potenzialmente accompagnati da determinate strutture in LM ma che, invece, in LO sono accompagnati da altre. Vediamo qui di seguito un esempio inerente alla perifrasi stare + gerundio, adatto per studenti di livello intermedio iniziale; il testo potrà essere opportunamente inquadrato in un'unità didattica adatta al tema e al suo livello di complessità:

\section{Una notte terribile}

La notte fra giovedi e venerdi ha piovuto senza sosta dalle 22 alle 5 del mattino. Come lo so? Lo so perché alle 10 del venerdi avevo la presentazione del mio progetto, il momento più importante dell'anno! Anche io, come la pioggia, non ho dormito nemmeno un minuto: ho lavorato tutta la sera e poi tutta la notte, fino alle 4, al testo, al power point, alle cose da dire, al discorso. Che ansia! Alle 4 sono andato a letto, stanchissimo. Ma al piano di sotto stavano facendo una festa: hanno ballato e gridato senza pausa per sette ore, fino alle 6, e cosi io non ho chiuso occhio. Poco dopo le 6 ho deciso di scendere a passeggiare per rilassarmi un po'. Il bar all'angolo era già aperto, e così sono entrato a bere un caffè. Al bancone accanto a me c'erano un uomo e una donna, anche loro con il caffè. Avevano l'aria stanca e parlavano a voce bassa. Mi hanno guardato e la donna mi ha detto: "è un lavoratore della notte anche Lei? Ha fatto come noi il turno di notte?". "In un certo senso sì", pensai: "sì, in effetti... in qualche modo si, ho scritto e ripetuto il mio progetto per tutta la notte". "Ah, ha un colloquio fra poco", mi disse l'uomo. "Buona fortuna, e vedrà che bella dormita farà quando tutto sarà finito!" 
Come è possibile notare, nel testo sono evidenziate in grassetto espressioni di tempo continuato, che in spagnolo potrebbero introdurre il valore continuativo di stare + gerundio, e sono sottolineati i verbi che in italiano si accompagnano a quelle espressioni, ovvero, in questo caso, semplici passati prossimi. Queste evidenziazioni potrebbero portare l'attenzione selettiva dello studente ispanofono a notare tali concomitanze e a chiedere all'insegnante il perché di quelle manipolazioni testuali. Il docente potrebbe, a questo punto, far notare che, mentre in spagnolo la perifrasi stare + gerundio si può usare in tali contesti continuativi al passato, in italiano, come emerge dalle evidenziazioni, tale uso non è possibile, innescando così il noticing di ciò che in italiano non appare in tali contesti e di quale è, invece, la struttura adeguata da usare.

Esercizi simili possono essere proposti per le perifrasi ir/andare e venir/venire + gerundio. In questi casi, occorre esemplificare bene il contesto diafasico e diacronico in cui in italiano (non) si usano tali perifrasi, facendo ad esempio ricorso a una risorsa iconica che collochi chiaramente un enunciato in un determinato contesto comunicativo o in una determinata dimensione temporale. Una frase come è da tempo che Maria viene raccontando questa cosa potrebbe essere messa in relazione, magari proprio in un fumetto, con un'immagine che mostra una situazione comunicativa molto formale o collocata nel passato; la frase è da tempo che Maria racconta questa cosa dovrebbe invece essere contestualizzata in una dimensione diafasica quotidiana e in uno spazio temporale contemporaneo. I discenti potrebbero essere spinti a osservare le due immagini e i relativi enunciati e, grazie all'aiuto dell'insegnante, potrebbe essere così favorita un'analisi comparativa dell'uso della perifrasi venire/ venir + gerundio, mostrandone i limiti di espansione a cui è soggetta in italiano.

Ipoteticamente, in una classe composta solo da ispanofoni, il confronto potrebbe essere corroborato dal paragone del testo italiano con una controparte in spagnolo, in modo da rendere ancora più efficace la visione contrastiva.

In generale, attività di input strutturato come quella proposta (e le sue tante possibili diverse declinazioni) aiutano gli apprendenti a esplorare le zone d'ombra dello stimolo linguistico e li guidano nella scoperta dell'esistenza di prove negative indirette su cui basare la ristrutturazione delle loro ipotesi interlinguistiche.

\subsection{Attività di ispirazione cognitiva}

Un ultimo suggerimento operativo che riteniamo utile estendere trae ispirazione dalla linguistica cognitiva, i cui principi si sono dimostrati risorsa efficace per facilitare l'apprendimento di costruzioni e strutture complesse inerenti alla codifica di relazioni spaziali e temporali. Secondo la linguistica cognitiva, infatti, le costruzioni grammaticali sono convenzionali e language-specific, e il loro apprendimento implica il riconoscimento e la comprensione dei meccanismi cognitivi loro sottostanti (Langacker 2008, cfr. anche §3.3). La stretta connessione tra apprendimento linguistico ed esperienza del mondo fisico, che determina il carattere usage-based del paradigma cognitivo, motiva inoltre la promozione dell'impiego di diagrammi, disegni e animazioni in una classe di lingua straniera. Tali accorgimenti didattici rendono discernibili, tramite un canale sensoriale più accessibile - quello visivo, appunto - i meccanismi cognitivi che motivano la morfosintassi, facilitandone la comprensione e la memorizzazione. 
36

Ambrosini, M. V.; Della Putta, P. Cuad. filol. ital. 28, 2021: 11-44

Uno studio che ha dato risultati interessanti sull'apprendimento della reale portata d'uso di stare + gerundio è presentato in Della Putta e Strik Lievers 2020. Tramite l'uso di alcuni semplici disegni, l'insegnante ha messo in risalto i diversi valori aspettuali che stare + gerundio ed estar + gerundio esprimono in italiano e spagnolo. Ne vediamo alcuni esempi nelle Figure 1 e 2.

Figura 1: aspetto continuativo e progressivo in spagnolo

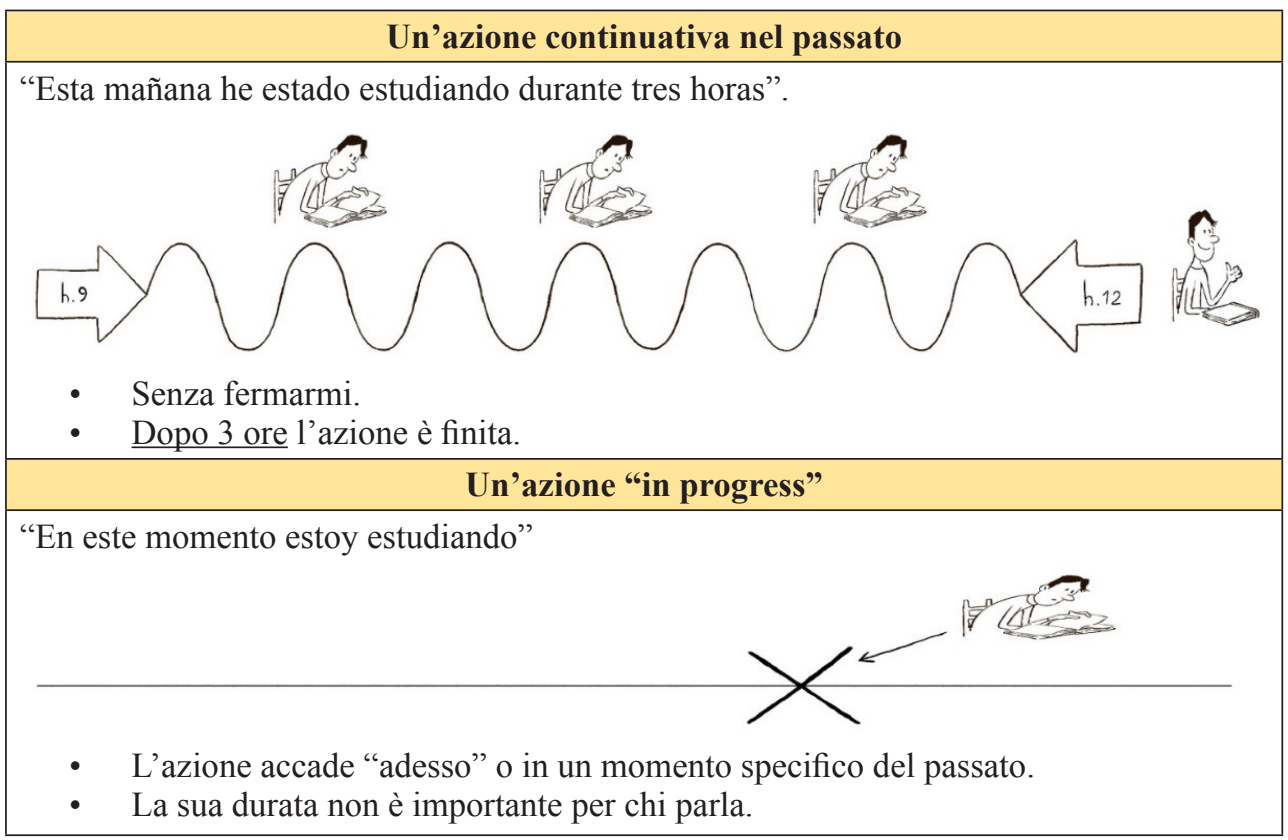

Figura 2: aspetto continuativo e progressivo in italiano

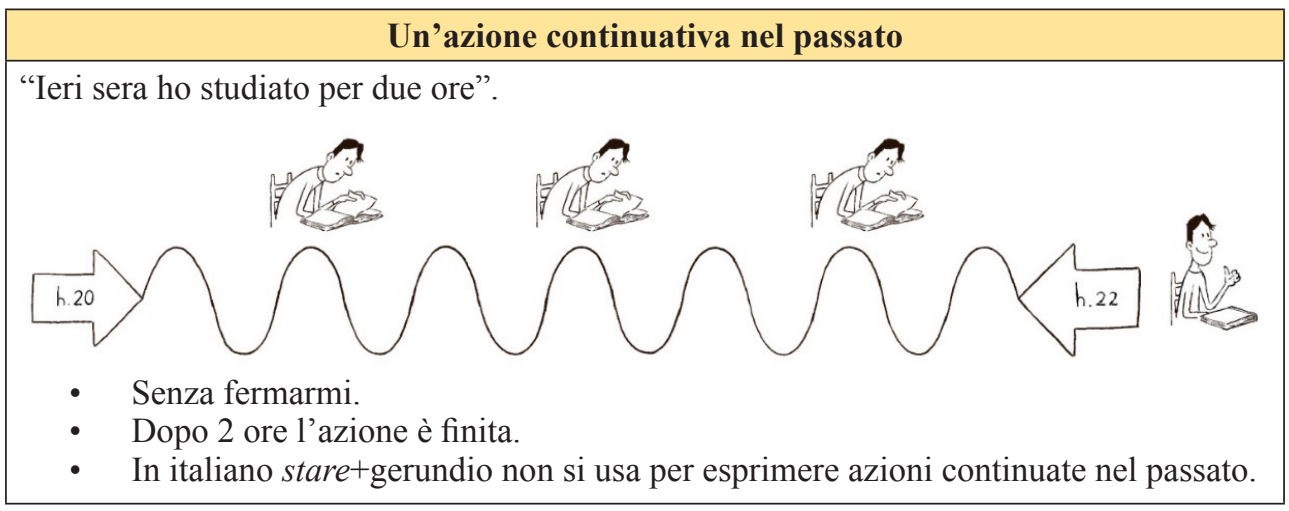


Un'azione "in progress"

"In questo momento sto studiando"

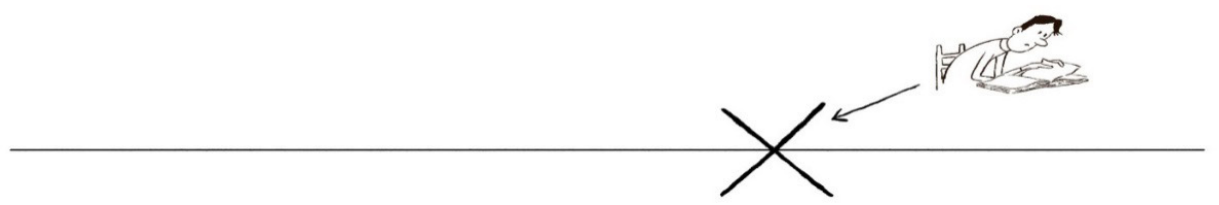

- L'azione accade "adesso" o in un momento specifico del passato.

- La sua durata non è importante per chi parla.

Grazie ai disegni, gli apprendenti hanno avuto l'opportunità di ragionare in primo luogo sulla differenza fra aspetto continuativo e progressivo e, in secondo luogo, sulle strutture perifrastiche e/o analitiche che italiano e spagnolo usano per esprimere tali valori aspettuali. L'analisi contrastiva è dunque supportata dalle immagini, che favoriscono una comprensione più profonda delle funzioni che le forme della LM e della LO esprimono. A queste prime attività, ripetute per alcune lezioni, sono seguite altre proposte didattiche più coinvolgenti e creative, come la richiesta di disegnare o schematizzare frasi o brevi brani in cui i diversi aspetti venivano narrati. L'attenzione dell'insegnante è stata mantenuta alta sulle discrepanze d'uso di stare/ estar + gerundio, e alcune delle mosse correttive/interattive viste in $\$ 5.1$ sono state effettivamente attuate. In seguito, sono stati proposti esercizi di transcodificazione immagine $\rightarrow$ testo, proposti come lavori di coppia o di gruppo. Gli studenti avevano il compito di descrivere con una frase o con una breve narrazione alcune immagini in serie che ritraevano scenari aspettuali simili a quelli delle Figure 1 e 2. Bisognava quindi in primo luogo esercitare una certa sensibilità aspettuale, cercando di capire se le azioni ritratte dalle immagini fossero continuative, progressive o semplicemente perfettive; in seguito, il compito di ogni gruppo era di trovare soluzioni linguistiche adatte per descrivere i disegni, attivando così la consapevolezza di quali sono i mezzi che l'italiano ha per esprimere valori aspettuali anche complessi. I risultati dello studio (a cui rimandiamo per dettagli) sono stati confortanti: non solo gli studenti hanno accettato di buon grado le proposte pedagogiche, ma i risultati di post test $\mathrm{e}$ delayed post test mostrano risultati d'apprendimento di buona portata.

Tali attività pittoriche e rappresentative sono state proposte a giovani studenti ispanofoni d'arte iscritti per circa un anno a un'accademia di belle arti italiana. È palese che questa categoria di studenti, maggiormente avvezza al disegno e all'astrazione, potrebbe ricevere meglio queste proposte rispetto a un gruppo di apprendenti più adulti o con altri interessi e inclinazioni culturali. Seguendo le riflessioni sul senso di plausibilità di ogni docente fatte in $\S 5$, ci sentiamo qui di consigliare accorgimenti didattici basati sulla semplificazione cognitiva e sull'esemplificazione visuale delle differenze che intercorrono fra italiano e spagnolo in merito alle perifrasi; ogni docente, però, potrà dare diverso peso a queste attività nel suo programma, o potrà trovare applicazioni diverse di questi principi a seconda della classe con cui si troverà a lavorare. 


\section{Conclusioni}

Nel presente lavoro, abbiamo inteso proporre un focus su due fenomeni di complessificazione riscontrabili nell'interlingua di apprendenti di italiano ispanofoni, generati da un transfer di addizione superflua di tre perifrasi della loro LM: ir + gerundio, venir + gerundio ed estar + gerundio.

Il primo di questi tre fenomeni di complessificazione riguarda le perifrasi ir/venir + gerundio: esso si verifica nel momento in cui l'apprendente utilizza le perifrasi italiane formalmente corrispondenti, andare/venire + gerundio, in contesti comunicativi informali, nei quali il loro uso non risulta adeguato; il secondo fenomeno, di matrice più prettamente morfosintattica, riguarda invece la perifrasi estar + gerundio: in questo caso, l'apprendente tende a mapparne, sul costrutto italiano corrispondente stare + gerundio, non solamente il valore aspettuale progressivo - veicolato anche dalla perifrasi italiana - ma anche quello continuativo - assente invece in quest'ultima.

Come abbiamo illustrato tramite i dati esposti in $\$ 3.3$, si tratta di fenomeni di complessificazione piuttosto diffusi nell'interlingua degli apprendenti di italiano ispanofoni, compresa quella di apprendenti di livello intermedio o avanzato. Abbiamo riflettuto su come sia difficile, per un apprendente ispanofono, rendersi consapevole di questi processi in termini di riflessione metalinguistica. A tal fine, egli dovrebbe giungere a notare che, in LO:

- Le perifrasi andare/venire + gerundio, al contrario di quanto accade per $\mathrm{ir} /$ venir + gerundio, non compaiono mai nelle varietà di registro informali, essendo invece utilizzate solamente in comunicazioni dal registro piuttosto elevato;

- stare + gerundio, al contrario della corrispondente spagnola estar + gerundio, non può veicolare l'aspetto continuativo e, conseguentemente, non ammette che l'ausiliare sia una forma verbale composta.

Come già esposto in $\S 5$, è piuttosto complesso proporre una strategia didattica che porti l'apprendente a maturare la consapevolezza di questi elementi di contrastività, tanto più se si considerano gli innegabili fattori di complicazione che incidono nel processo di ristrutturazione interlinguistica inerente a queste tre perifrasi: le complessità psicolinguistiche di ristrutturazione viste in $\S 2$ e $\$ 3.3$, il livello di opacità ed esiguità delle prove negative (dirette e indirette) che lo studente riceve in termini di input dalla LO, il carente supporto nozionale offerto dalle grammatiche attualmente in uso, che è insufficiente a indurre nell'apprendente riflessioni che siano davvero efficaci a livello contrastivo (cfr §4). Questi presupposti rendono l'inibizione delle tipologie di transfer analizzate in questo contributo estremamente complessa: il docente che si proponga di venirne a capo dovrebbe dunque porre particolare cura e consapevolezza nel proporre una formula pedagogica che porti l'apprendente ad attivare un atteggiamento strategicamente contrastivo nello studio e nell'assimilazione delle tre perifrasi italiane coinvolte, facendo leva sulle asimmetrie che ne caratterizzano il rapporto con le corrispondenti spagnole e neutralizzando così le pressioni fusionali date dall'affinità tra LM e LO.

La scelta di includere nel presente contributo, accanto al raffronto tra estar + gerundio/stare + gerundio anche quello tra $\mathrm{ir} / v e n i r+$ gerundio e andare/venire + gerundio, ci ha permesso di mostrare come il transfer non scaturisca sempre e solo da 
una divergenza di matrice morfosintattica tra LM e LO: esso può anche manifestarsi nel caso di due strutture che - seppur formalmente corrispondenti tra le due lingue non vengono da esse impiegate in modo o distribuzione corrispondenti nei rispettivi repertori varietistici. È questo il caso della divergenza tra ir/venir + gerundio e andare/venire + gerundio. Tale divergenza, pur non dando luogo a errori grammaticali come nel caso di estar/stare + gerundio, può comunque inficiare in modo consistente l'adeguatezza comunicativa dell'apprendente, conseguenza che potrebbe al più essere tollerata nei livelli di competenza più bassi, ma dovrebbe auspicabilmente non riscontrarsi in quelli più avanzati. A tal proposito, sono piuttosto pertinenti le osservazioni di Benucci (2015: 267):

Sul piano pragmatico, l'italiano degli stranieri (parlato ma anche scritto) non si avvicina per espressività e incisività a quello dei nativi. [...] Delle tre cause ipotizzabili:

1. non conoscenza delle regole;

2. difficoltà interne del sistema italiano;

3. assenza di una visione della competenza comunicativa che tenga conto in eguale misura delle sue due principali componenti, quella linguistica e quella pragmatica; la seconda e la terza sono probabilmente le più significative. Infatti, possiamo affermare con certezza che chi studia l'italiano L2 sia stato sensibilizzato rispetto al complesso problema della variazione e sia in possesso degli strumenti per ottenere produzioni quanto più possibile vicine a quelle dei nativi, cioè possieda assieme a una visione plurilinguistica anche una visione pluriculturale dell'Italia? I programmi, i materiali e i libri di testo danno la possibilità di formarsi un tale tipo di competenza?

Proposte didattiche come quelle che abbiamo illustrato in $\S 5.1$ e $\S 5.2$ nascono in risposta all'esigenza di una didattica sensibile e reattiva a queste dinamiche.

Un obiettivo ambizioso ma ineludibile per il docente che si proponga di creare un percorso didattico produttivamente contrastivo e che aspiri non tanto e non solo all'insegnamento di un italiano corretto, ma anche all'insegnamento di un italiano reale.

\section{Bibliografia}

Abutalebi, Jubin / Della Rosa, Pasquale / Ding, Guosheng / Weekes, Brendan / Costa, Albert / Green, David (2013): «Language proficiency modulates the engagement of cognitive control areas in multilinguals», Cortex, 49, pp. 305-911.

Ambrosini, Maria Vittoria (2018): La expresión de la modalidad continuativa en italiano, español y catalán: Analogías, divergencias e interferencias, Roma, Aracne Editrice.

Ambrosini, Maria Vittoria (2020): «Il rapporto tra STARE + GERUNDIO/ESTAR + GERUNDIO nella didattica dell'italiano per ispanofoni: una questione di contrastività», in G. Caprara, G. Marangon (a c. di), Italiano y español. Estudios de traducción, lingüística contrastiva y didáctica, Frankfurt am Main/Bern: Peter Lang GmbH, pp. 425-438.

Amenós-Pons, José / Ahern, Aoife / Guijarro-Fuentes, Pedro (2017): «L1 French learning of L2 Spanish past tenses: L1 transfer versus aspect and interface issues», Studies in Second Language Learning and Teaching, 7, pp. 789-515. 
Bailini, Sonia (2016): La interlengua de lenguas afines. El español de los italianos, el italiano de los españoles, Milano, LED.

Bailini, Sonia / Frigerio, Aldo (2018): «CORESPI e CORITE, due nuovi strumenti per l'analisi dell'interlingua di lingue affini», CHIMERA: Romance Corpora and Linguistic Studies, 5, pp. 313-319.

Barbagallo, Simona (2014): «Estudio contrastivo de algunas perífrasis de gerundio en español e italiano», Annali della facoltà di scienze della formazione, 13, pp. 179-197.

Benazzo, Sandra / Andorno, Cecilia (2017): «Is it really easier to acquire a closely-related language? A study on the expression of iteration and continuation in L2 French», in H. Martin, P. Leclercq, (a c. di), Tense-Aspect-Modality in a Second Language, Amsterdam, John Benjamins, pp. 105-143.

Benucci, Antonella (a cura di) (2007): Sillabo di italiano per stranieri, Perugia, Guerra.

Benucci, Antonella (2015): «La correzione degli errori in italiano L2», in P. Diadori (a c. di), Insegnare l'italiano a stranieri, Milano, Mondadori Education S.p.A.

Benso, Francesco (2013): «Sistema attentivo esecutivo: osservazioni critiche e sviluppo», in L. Sabbadini (a c. di), Disturbi specifici del linguaggio, disprassie e funzioni esecutive, Milano, Springer, pp. 41-84.

Bertinetto, Pier Marco (1986): Tempo, Aspetto e Azione nel verbo italiano. Il sistema dell'indicativo, Firenze, Accademia della Crusca.

Bertinetto, Pier Marco (1991): «Il verbo», in A. Cardinaletti (a c. di), Grande Grammatica italiana di consultazione, Bologna il Mulino, vol. 2, pp. 13-161.

Bertinetto, Pier Marco (1997): Il Dominio tempo-aspettuale, Torino, Rosemberg e Sellier.

Bertinetto, Pier Marco (2000): «The progressive in Romance, as compared with English», in Ö. Dahl (a c. di), Tense and Aspect in the Languages of Europe, Mouton de Gruyter, pp. 559-604.

Blasco Ibañez, Vicente (2008): El paraíso de las mujeres, Barcelona, Libros Mablaz.

Brianti, Giovanna (2000): «Diacronia delle perifrasi aspettuali dell'italiano: Il caso di "stare" + gerundio, "andare" e "venire" + gerundio», Lingua Nostra, 61, pp. 35-52; 97-120.

Brianti, Giovanna (2010): «La perifrasi progressiva 'in progress': confronto tra italiano e inglese», in M. A. Terzoli, R. A. Asor, G. Inglese (a c. di), Letteratura e filologia fra Svizzera e Italia. Studi in onore di Guglielmo Gorni, vol. III. Dall'Ottocento al Novecento: letteratura e linguistica, Roma, Edizioni di storia e letteratura, pp. 351-362.

Bruton, Anthony (2000): «What exactly are positive and negative evidence in SLA?», RELC Journal, 2, pp. 120-133.

Caddeo, Roberta (2019): «Aspetti dell'interferenza italiano-sardo: il gerundio nell'italiano regionale di Sardegna», Rhesis. International Journal of Linguistics, Philology and Literature, 10 , pp. $75-112$.

Calvi, Maria Vittoria (1995): Didattica di lingue affini. Spagnolo e italiano, Milano, Guerini scientifica.

Camus Bergareche, Bruno (2004): «Perífrasis verbales y expresión del aspecto en español», in L. García Fernández, B. Camus Bergareche (a c. di), El pretérito imperfecto, Madrid, Gredos, pp. 511-572.

Carrera Díaz, Manuel (1979): «Italiano para hispanohablantes: la engañosa facilidad», Rassegna Italiana di Linguistica Applicata, 11, pp. 23-40.

Carrera Díaz, Manuel (1992): Curso de lengua italiana. Parte teórica, Barcelona, Ariel.

Carrera Díaz, Manuel ([1985] 2015): Manual de gramática italiana, Barcelona, Ariel

Corder, Pit (1984): «La lingua dell'apprendente», in E. Arcaini, B. Py (a c. di.), Interlingua, aspetti teorici e implicazioni didattiche, Roma, Istituto della Enciclopedia Italiana, pp. 49-72. 
Daloiso, Michele (2018): «La spiegazione della perifrasi progressiva nelle grammatiche d'italiano per stranieri: analisi di alcuni testi e proposte glottodidattiche ispirate alla Linguistica Cognitiva», Italiano Linguadue, 10, pp. 1-24.

Della Putta, Paolo (2015): «How to discourage constructional negative transfer: Theoretical aspects and classroom activities», in K. Masuda, C. Arnett, A. Labarca (eds.), Cognitive Grammar and Sociocultural Theory in Foreign and Second Language Teaching, Berlin, Mouton de Gruyter, pp. 25-50.

Della Putta, Paolo (2016), «Do we also need to unlearn constructions? The case of constructional negative transfer from Spanish to Italian and its pedagogical implications», in S. De Knop, G. Gilquin (eds.), Applied Construction Grammar, Berlin, Mouton de Gruyter, pp. 237-269.

Della Putta, Paolo (2018): «Enhancing nothing? The efficacy of textual enhancement in fostering the learning and the unlearning of two syntactic properties of Spanish and Italian», in SILTA - Studi Italiani di Linguistica Teorica e Applicata, vol. XLVII, pp. 239-256.

Della Putta, Paolo / Strik Lievers, Francesca (2020): «From Spanish <estar + gerund > to Italian $<$ stare + gerund $>$. When teaching to unlearn is needed», Zeitschrift fuer Interkulurelles Sprachenunterricht, 25, pp. 1407-1430.

Diamond, Adele (2013): «Executive Functions», Annual Review of Psychology, 64, pp. 135168.

Díaz, Lourdes / Bel, Aurora / Bekiou, Konstantina (2007): «The role of morphological features in the acquisition of Spanish aspectual differences», in J. Liceras, H. Zobl, H. Goodluck (eds.), The Role of Formal Features in Second Language Acquisition, Mahwah, Lawrence Erlbaum, pp. 485-511.

Ellis, Nick (2006): «Selective attention and transfer phenomena in L2 acquisition: contingency, cue competition, salience, interference, overshadowing, blocking, and perceptual learning», Applied Linguistics, 27, pp. 164-194.

Ellis, Nick (2019): «Essentials of a theory of language $C_{j}$ cognition», The Modern Language Journal, 103, pp. 36-90.

Ellis, Nick / Sagarra, Nuria (2010): «The bounds of adult language acquisition: blocking and learned attention», Studies in Second Language Acquisition, 32, pp. 553-580.

Fernández de Castro, Félix (2000): Las perifrasis verbales en el español actual, Madrid, Gredos.

García Fernández, Luis (ed.) / Carrasco Gutiérrez, Ángeles / Camus Bergareche, Bruno / Martínez Atienza, María / García Serrano, María de los Ángeles (2006): Diccionario de perifrasis verbales, Madrid, Gredos.

García Fernández, Luis / Krivochen, Diego Gabriel (2019): Las perifrasis verbales en contraste, Madrid, Arco Libros.

Gass, Susan (1997): Input, Interaction, and the Second Language Learner, Mahwah, Erlbaum.

Giacalone Ramat, Anna (1995): «Sulla grammatizzazione dei verbi di movimento “andare” e "venire" + gerundio», Archivio glottologico italiano, 80, pp. 168-203.

Goldberg, Adele (2003): «Constructions: A new theoretical approach to language», Trends in Cognitive Sciences, 7, pp. 219-224.

Gómez Torrego, Leonardo (1999): «Los verbos auxiliares. Las perífrasis verbales de infinitivo», in I. Bosque, V. Demonte (eds.), Gramática descriptiva de la lengua española, Madrid, Espasa Calpe, pp. 3323-3390.

Housen, Alex / De Clerq, Bastien / Kuiken, Folkert / Vedder, Inneke (2019): «Multiple approaches to complexity in second language research», Second Language Research, 35, pp. 3-21. 
Jarvis, Scott (2012): «The detection-based approach: An overview», in S. Jarvis, S. Crosley (eds.), Approaching language transfer through text classification, Bristol, Multilingual Matters, pp. 1-34.

Jarvis, Scott / O’Malley, Michelle / Jing, Linye / Zhang, Jing / Hill, Jessica / Chan, Curtis / Sevostyanova, Nadezhede (2013): «Cognitive foundations of crosslinguistic influence», in J. Schwieter (ed.), Innovative Research and Practices in Second Language Acquisition and Bilingualism, Amsterdam, Benjamins, pp. 287-307.

Langacker, Ronald (1988): «An overview of Cognitive Grammar», in B. Rudzka-Ostyn (ed.), Topics in Cognitive Linguistics, Amsterdam, Benjamins, pp. 3-47.

Langacker, Ronald (2008): Cognitive Grammar. Oxford, OUP.

Lavinio, Cristina (2018): «Educazione linguistica e italiani regionali», in S. Gensini, M. E. Piemontese, G. Solimine (a c. di), Tullio De Mauro. Un intellettuale italiano, Roma, Sapienza Università Editrice, pp. 115-126.

Linck, Jared / Schwieter, John / Sunderman, Gretchen (2020): «The differential role of executive functions in the cognitive control of language switching», Languages, 5, pp. 1-19.

Lo Duca, Maria Giuseppa (2006): Sillabo di italiano L2, Roma, Carocci.

Martínez-Atienza, María (2004): «La expresión de la habitualidad en español», in L. García Fernández, B. Camus Bergareche (eds.), El Pretérito imperfecto, Madrid, Gredos, pp. 346-377.

Martínez-Atienza, María (2017): «Venir + gerundio del español, venire + gerundio del italiano y otras perífrasis verbales», Sintagma: revista de lingüística, 29, p. 27-42. https:// doi. org/10.21001/sintagma.2017.29.02.

Masini, Francesca (2016): Grammatica delle costruzioni, Roma, Carocci.

Montserrat, i Buendia, Sandra (2005): El canvi semàntic en la llengua antiga i medieval: el cas de la perifrasi $<$ estar $+G E R>$, projecte d'investigació original presentat a la Universitat de Barcelona el 7 de setembre de 2005, en el marc de les proves d'habilitació nacional que faculten per a concórrer a concurs d'accessos al cos de Professors Titulars d'Universitat.

Moretti, Giovanni Battista / Orvieto, Giorgio Raffaele (1979): Grammatica Italiana, vol. 2, Il verbo, Perugia, Benucci.

Morgana, Silvia / Zaffaroni, Anna (2011): «L'insegnamento dell'italiano L2 a ispanofoni. Aspetti e proposte didattiche», in M.V. Calvi, G. Mapelli, M. Bonomi (a c. di), Lingua, identità e immigrazione. Prospettive interdisciplinari, Milano, Franco Angeli, pp. 191208.

Musto, Salvatore / Ripa, Valentina (2005): «Le perifrasi gerundivali in spagnolo e in italiano: uno studio contrastivo», in M. C. Ruta, L. Silvestri (a c. di), Actas XXIII Congreso AISPI, Palermo, Flaccovio Editore, pp. 408-427.

Myers, Marie (2004): Modalités d'apprentissage d'une langue seconde, Louvain-la-Neuve, De Boeck Supérieur.

Odlin, Terence (2008): «Conceptual transfer and meaning extension», in P. Robinson, N. Ellis (eds.), Handbook of Cognitive Linguistics and Second Language Acquisition, New York / London, Routledge, pp. 306-340.

Olbertz, Hella (1998): Verbal Periphrases in a Functional Grammar of Spanish, Berlin / New York, Mouton de Gruyter.

Olesen, Lone (2010): «Italiano e sardo in contatto: italiano regionale o sardo italianizzato?», in J. Havu, C. Klippi, S. Hakulinen, J. Philippe, J. Santisteban (éds.), Actes du XVIIe congrès des romanistes scandinaves, Tampere, Tampere University Press, pp. 932-954. 
Oricco, Matilde (2020): Analisi di quattro asimmetrie strutturali fra italiano e spagnolo in alcune grammatiche di italiano per ispanofoni, Tesi di laurea non pubblicata, Università di Torino.

Peters, Elke / Muñoz, Carmen (2020): «Introduction to Special Issue Language Learning from Multimodal Input», Studies in Second Language Acquisition, 42, pp. 489-497.

RAE-ASALE (=Real Academia Española / Asociación de Academias le la Lengua Española (2009): Nueva gramática de la lengua española, Madrid, Espasa Calpe.

Ringbom, Hakan (2007): Cross-linguistic Similarity in Foreign Language Learning, Clevedon, Multilingual Matters.

Ringbom, Hakan / Jarvis, Scott (2009): «The importance of cross-linguistic similarity in foreign language learning», in M. Long, C. Doughty (eds.), The Handbook of Language Teaching, New York, Blackwell, pp. 106-118.

Robenhalt, Clarice / Goldberg, Adele (2016): «L2 learners do not take competing alternative expressions into account the way L1 learners do», Language Learning, 66, pp. 60-93.

Rosi, Fabiana (2020): «Intercomprensione e plurilinguismo: studenti universitari di lingue e di discipline non linguistiche a confronto», Cuadernos de filología italiana, 27, pp. 137165.

Sánchez Montero, Mª Del Carmen (1993): Perífrasis verbales en español e italiano. Estudio contrastivo, Trieste, Edizioni Lint.

Schmid, Stefan (1994): L'italiano degli spagnoli. Interlingue di immigrati nella svizzera tedesca, Milano, Franco Angeli.

Schmid, Stephan (2015): «Italiano e spagnolo in contatto: ibridazione linguistica e trasferibilità di morfemi legati in due lingue imparentate», in L. Filipponio, C. Seidl (a c. di), Le lingue d'Italia e le altre, Milano, Franco Angeli, pp. 249-270.

Spada, Nina (2019): «Discussion: balancing methodological rigor and pedagogical relevance», in R. Dekeyser, B. Prieto Goretti (eds.), Doing SLA Research with Implications for the Classroom, Amsterdam, Benjamins, pp. 201-215.

Squartini, Mario (1998): Verbal Periphrases in Romance: Aspect, Actionality, and Grammaticalization, Berlin/New York, Mouton de Gruyter.

Trovato, Giuseppe (2014): «Las perífrasis verbales en contrastividad entre español e italiano: perspectiva metalingüística y traductológica», AGON (Rivista Internazionale di Studi Letterari, Linguistici e Culturali), 2, pp. 37-70.

Ur, Penny (2019): «Theory and practice in language teacher education», Language Teaching, 52, pp. 450-459.

Valentini, Ada (2016): «La prospettiva psicolinguistica sull'input in L2», in A. Valentini (a c. di), L'input per l'acquisizione di lingue seconde: strutturazione, percezione, elaborazione, Firenze, Cesati, pp. 25-42.

Vendler, Zeno (1967): Linguistics in Philosophy, Cornell University Press, Ithaca.

Vietti, Alessandro (2005): Come gli immigrati cambiano l'italiano. L'italiano di peruviane come varietà etnica, Milano, Franco Angeli.

Vietti, Alessandro (2010): «Italiano e spagnolo a contatto: immigrazione e varietà etnica», in M.V. Calvi, G. Mapelli, M. Bonomi (a c. di), Lingua, identità e migrazione. Prospettive interdisciplinari, Milano, Franco Angeli, pp. 221-236.

Yllera, Alicia (1999): «Las perífrasis verbales de gerundio y participio», in I. Bosque, V. Demonte (eds.), Gramática descriptiva de la lengua española, Madrid, Espasa Calpe, pp. 3391-3441. 


\section{Sitografia}

Calleja, T. (2006): Murcia recurrirá el traspaso del Guadalquivir. elpais.com, https://elpais. com/diario/2006/05/14/andalucia/1147558924_850215.html [21/04/2021].

foros.abc.com, commento del 05/03/2015, http://foros.acb.com/viewtopic.php?t=484245 \&start $=145760$ [21/04/2021].

Cárdenas Utrilla, L. (2020): Reinaldo Rueda: «Hace Años que Colombia viene siendo un equipo muy competitivo». marcaClaro.com, https://co.marca.com/claro/futbol/seleccion-colombia/2020/10/12/5f8492bd46163fd3be8b458d.html [21/04/2021].

S.Font, V. (2009): Cuando la guerra se lleva a cabo con fármacos. elmundo.es Salud, http:// www.elmundo.es/elmundosalud/2009/08/19/biociencia/1250704184.html [21/04/2021].

LaCosaRosa.com, 25/09/2016, http://www.lacosarosa.com/brad-pitt-considera-ambicionespoliticas-angelina-jolie-perjudicaran-hijos.html [21/04/2021]. 